\title{
The chirality-flow formalism
}

\author{
Andrew Lifson $^{\mathrm{a}}$, Christian Reuschle ${ }^{\mathrm{b}}$, Malin Sjodahl ${ }^{\mathrm{c}}$ \\ Department of Astronomy and Theoretical Physics, Lund University, Sölvegatan 14A, 22362 Lund, Sweden
}

Received: 9 July 2020 / Accepted: 18 July 2020 / Published online: 30 October 2020

(C) The Author(s) 2020

\begin{abstract}
We take a fresh look at Feynman diagrams in the spinor-helicity formalism. Focusing on tree-level massless QED and QCD, we develop a new and conceptually simple graphical method for their calculation. In this pictorial method, which we dub the chirality-flow formalism, Feynman diagrams are directly represented in terms of chiralityflow lines corresponding to spinor inner products, without the need to resort to intermediate algebraic manipulations.
\end{abstract}

\section{Contents}

1 Introduction . . . . . . . . . . . . . . 1

2 Color flow ............... 2

3 The basics of the spinor-helicity formalism . . . . 3

3.1 Spinors and spinor inner products . . . . . . 3

3.2 Four-vectors . . . . . . . . . . . . . . . 4

3.3 Polarization vectors . . . . . . . . . . 5

4 Building the chirality-flow picture . . . . . . . 6

4.1 A simple QED example . . . . . . . . . . 6

4.2 Proof for QED . . . . . . . . . . . . . 8

4.3 Proof for QCD . . . . . . . . . . . . . . . . . 8

4.4 QCD remarks ............ . . . . . 8

5 Chirality-flow Feynman rules . . . . . . . . . . 9

5.1 Vertices .................... 9

5.2 Propagators ................... 11

5.3 Application ................ 12

6 Examples ....................... 12

$6.1 e^{+} e^{-} \rightarrow \mu^{+} \mu^{-} \ldots \ldots . . . \ldots 13$

$6.2 e^{+} e^{-} \rightarrow \mu^{+} \mu^{-} \gamma \ldots \ldots . \ldots . . \ldots 13$

$6.3 q_{1} \bar{q}_{1} \rightarrow q_{2} \bar{q}_{2}$..................... 15

$6.4 q \bar{q} \rightarrow g g \ldots \ldots . \ldots . . \ldots 18$

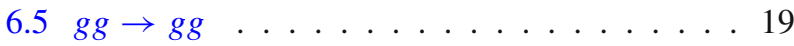

7 Conclusion and outlook ........... . 21

\footnotetext{
a e-mail: andrew.lifson@thep.lu.se

b e-mail: christian.reuschle@ thep.lu.se

c e-mail: malin.sjodahl@thep.lu.se (corresponding author)
}

A Conventions and identities . . . . . . . . . 21

A.1 Pauli matrices . . . . . . . . . . . . 21

A.2 Spinors and spinor inner products . . . . . . 22

A.3 Four-vectors and bispinors . . . . . . . . . . 23

A.4 Tables with QED and QCD conventions and Feynman rules . . . . . . . . . . . . . . . . 24

References . . . . . . . . . . . . . . . 26

\section{Introduction}

During the past decades, various techniques for calculating scattering amplitudes have emerged, resulting in both compact analytic formulae and efficient numerical approaches. In this context, strategies for managing quantum numbers, such as color and helicity have played a major role. Both for color and helicity, factorized approaches, where amplitudes have a particular color structure or helicity assignment, have been used.

In color decomposition, amplitudes are decomposed into color factors multiplied by smaller, gauge-invariant pieces, so-called partial amplitudes, and various approaches exist, differing in the way of choosing the set of vectors in which the color decomposition is obtained [1-32]. For helicity aplitudes, i.e. amplitudes with assigned helicities, and in particular for partial amplitudes, the spinor-helicity formalism in which diagrams and amplitudes are expressed in terms of two-component Weyl spinors, has been very successful [3348], especially after realizing that also the polarization vectors of external vector bosons can be expressed in terms of two-component Weyl spinors. In the Weyl-van-der-Waerden formalism [7, 8, 13-15,20,49-51], diagrams and amplitudes can even be expressed in such a way as to avoid Lorentz four-vectors entirely, using that Dirac spinors and Lorentz four-vectors transform under the $(1 / 2,0) \oplus(0,1 / 2)$ and $(1 / 2,1 / 2)=(1 / 2,0) \otimes(0,1 / 2)$ representations of the Lorentz group respectively. This is a fact which we also rely on in this paper. 
For diagrams or amplitudes in the spinor-helicity formalism, particularly compact analytic expressions exist in the form of the Parke-Taylor formula and other maximallyhelicity-violating (MHV) amplitudes [8,52-55]. However, also helicity amplitudes with more complicated next-tomaximally-helicity-violating (NMHV) configurations, etc., have been studied.

In the spinor-helicity formalism, calculations of single Feynman diagrams, as well as complete scattering amplitudes, are significantly simplified by expressing them in terms of spinor inner products. In this paper, we take a fresh look at Feynman diagrams in the spinor-helicity formalism in massless QED and QCD. In an attempt to further simplify their calculations, we extend the spinor-helicity formalism with an intuitive pictorial representation, reminiscent of the pictorial representations often used in the treatment of color degrees of freedom.

In the context of the color-flow picture, a graphical representation which provides an intuitive approach in terms of the flow of color is used $[3,19,20,28,32]$. Here, indices in the adjoint representation are converted to (pairs of) indices in the fundamental representation of $\mathrm{SU}(N)$ color, and the color degrees-of-freedom are accounted for by considering all possible connections in the space of fundamental color indices, i.e. all possible color flows. As an example, in a condensed notation, the four-gluon vertex can be expressed as

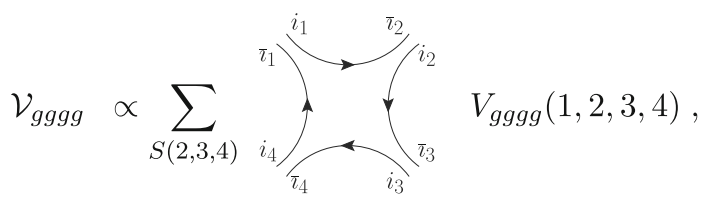

where $V_{\text {gggg }}(1,2,3,4)$ denotes the color-stripped four-gluon vertex in the color-flow picture, $S(2,3,4)$ denotes the set of permutations of the integers 2,3,4, and the lines represent the color flows.

Noting that the Lorentz group algebra consists of two copies of the (complexified) su(2) algebra, it can be anticipated that an analogous graphical approach should be applicable for the Lorentz group. ${ }^{1}$ Unlike the single su(3) color algebra, this would require two different types of flow lines - one dotted and one undotted - which can never be contracted into each other, since the corresponding object would not be Lorentz invariant. We dub this graphical approach the chirality-flow formalism.

More concretely, the chirality-flow formalism relies on the fact that objects carrying Lorentz indices can be con-

\footnotetext{
${ }^{1}$ Based on the Weyl-van-der-Waerden formalism, a double-line notation analogous to the double-line notation in the color-flow picture for spinor-helicity diagrams has been observed before [20]. A birdtrack $[4,56]$ based graphical formalism has also been noted [57]. However, to the best of our knowledge, a corresponding pictorial representation, with a directed continuous flow has not previously been formulated.
}

verted to objects carrying spinor indices instead. Feynman diagrams can then be rewritten in terms of contractions of spinor indices in a flow-like picture, similar to the color-flow picture. For example, the four-gluon vertex can be expressed in a similarly condensed notation as

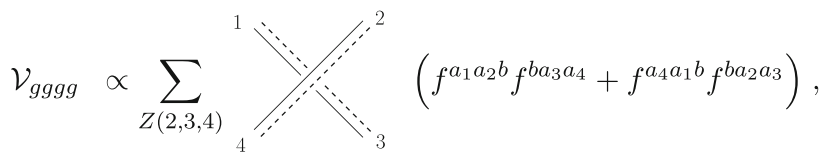

where $Z(2,3,4)$ denotes the set of cyclic permutations of the integers 2, 3, 4, and the lines represent the chirality flows.

In this new graphical formalism, the contractions of the spinor indices in Feynman diagrams are more obvious, allowing for a more transparent, shorter journey from Feynman diagrams to spinor inner products. The usual algebraic identities needed to transform a Feynman diagram into a set of spinor inner products are directly built into the pictorial representation.

We intend for this paper to serve as a readable introduction for beginners to the spinor-helicity formalism, and in this spirit we have written it in a self-contained manner, although some basic identities are only given in Appendix A. Further introductions and overviews can be found in [20,51,58-66].

The rest of the paper is organized as follows. In Sect. 2 we warm up by reviewing the color-flow idea. The traditional spinor-helicity formalism, as well as chirality-flow representations of external particles, inner products and slashed momenta are introduced in Sect. 3. Section 4 paves the way for the chirality-flow Feynman rules, as we complete and prove the validity of the chirality-flow picture for Feynman diagrams in massless QED and QCD. The Feynman rules are then collected in Sect. 5. In Sect. 6 we give examples, and in Sect. 7 we summarize and conclude.

\section{Color flow}

As a warm-up, let us start with considering a well-known example of a flow-like representation in the context of $\operatorname{SU}(N)$ scattering amplitudes - color flow (with $N$ colors).

In the color-flow formalism [3,19,20,28,32] the color factors of Feynman rules are converted into color-flow rules. Color indices in the adjoint representation of $\mathrm{SU}(N)$ are thereby converted to pairs of color indices, one in the fundamental representation and one in the antifundamental representation, and color factors are given by Kronecker $\delta$ 's, connecting the fundamental index of one parton to the antifundamental index of another parton. In other words, we can write the Fierz identity for the $\mathrm{SU}(N)$ generators

$t_{i j}^{a} t_{k l}^{a}=\delta_{i l} \delta_{k j}-\frac{1}{N} \delta_{i j} \delta_{k l}$, 
in a graphical representation

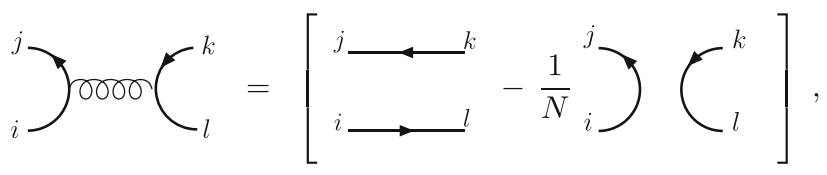

where the adjoint representation is replaced by (a linear combination of) flows of color.

Equation (2.2) can be seen as an example of color flow in the case of a gluon exchanged between two quark lines, in which case one also talks about the exchange of a $\mathrm{U}(N)$ gluon in the first term, and a U(1) gluon in the second, colorsuppressed term.

For gluon exchange between a quark line and a gluon, or in pure gluon interactions, the color-suppressed terms drop out, and it is possible to express gluons as $\mathrm{U}(N)$ gluons only. For example, the color flows of the four-gluon vertex are given in (1.1).

Given a diagram or amplitude one can write down all possible flows of color, and from these infer the corresponding color-stripped amplitudes or diagrams. The color-stripped Feynman rules in the color-flow formalism, sometimes also referred to as the double-line formalism, come with the benefit that diagrams and amplitudes are easier to calculate and can be given a more intuitive interpretation. ${ }^{2}$ In this paper we define a similarly intuitive picture, which we dub the chirality-flow formalism, for Feynman diagrams in the spinor-helicity formalism.

For future reference we define the generators $t$ and structure constants $f$ of the $\mathrm{SU}(N)$ color algebra by

$\operatorname{Tr}\left(t^{a} t^{b}\right)=\delta^{a b}$,
if $f^{a b c}=\operatorname{Tr}\left(t^{a}\left[t^{b}, t^{c}\right]\right)$,

with the indices $a, b, c=1, \ldots, N^{2}-1$ in the adjoint representation of $\operatorname{SU}(N)$, and the indices $i, j, k, l=1, \ldots, N$ in the (anti)fundamental representation. We normalize the generators to unity in (2.3) to avoid carrying around unnecessary powers of 2 in QCD algebra relations. This fixes the constant on the right-hand side of (2.1) to unity. Combining (2.3) with the algebra relation $\left[t^{a}, t^{b}\right]=i f^{a b c} t^{c}$, (2.4) follows.

\footnotetext{
2 Various approaches for color decomposition of QCD amplitudes, or $\mathrm{SU}(N)$ amplitudes in general, exist, differing in the way of choosing the set of vectors in which the color decomposition is obtained. The trace $[2,7-10,12-17,21,23,24,26-28,67-70]$ and color-flow $[3,19,20$, $28,32]$ representations have been very useful for deriving amplitudes, and analytic formulae exist for many cases [8-10,12-17,26-28]. On the other hand, orthogonal, group-theory based multiplet bases [1,4$6,11,22,25,29-31,56,69,71-73]$ are superior for squaring amplitudes, and adjoint (DDM) bases [18] may be advantageous for the pure gluon case.
}

\section{The basics of the spinor-helicity formalism}

In this section we review some basics of the spinor-helicity formalism, focusing on massless fermions and vector bosons (some additional relations are given in Appendix A, and more detailed introductions can be found in [20,51,58-66]). We also introduce the chirality-flow representations of spinors, spinor inner products and bispinors.

\subsection{Spinors and spinor inner products}

Let us first consider an incoming fermion or outgoing antifermion of momentum $p$ in the chiral, or Weyl, representation. For massless fermions and in a condensed notation we can write the corresponding four-component spinors in momentum space as

$u(p)=\left(\begin{array}{c}u_{L} \\ u_{R}\end{array}\right)=\left(\begin{array}{c}\tilde{\lambda}_{p}^{\dot{\alpha}} \\ \lambda_{p, \beta}\end{array}\right), \quad v(p)=\left(\begin{array}{c}v_{L} \\ v_{R}\end{array}\right)=\left(\begin{array}{c}\tilde{\lambda}_{p}^{\dot{\alpha}} \\ \lambda_{p, \beta}\end{array}\right)$,

where we have introduced the two-component Weyl spinors ${ }^{3}$ $\lambda_{p, \beta}$ and $\tilde{\lambda}_{p}^{\dot{\alpha}}$. The state $\tilde{\lambda}_{p}^{\dot{\alpha}}$, with a dotted index, transforms under the left-chiral $\left(\frac{1}{2}, 0\right)$-representation of the Lorentz group, while the state $\lambda_{p, \beta}$, with an undotted index, transforms under the right-chiral $\left(0, \frac{1}{2}\right)$-representation (see Appendix A.2). These states are projected out from the fourcomponent spinors by the chiral projection operators $P_{R / L}=$ $\frac{1}{2}\left(1+/-\gamma^{5}\right)$, such that for example $P_{L} v(p)=\left(\begin{array}{c}v_{L} \\ 0\end{array}\right)=\left(\begin{array}{c}\tilde{\lambda}_{p}^{\dot{\alpha}} \\ 0\end{array}\right)$ and $P_{R} v(p)=\left(\begin{array}{c}0 \\ v_{R}\end{array}\right)=\left(\begin{array}{c}0 \\ \lambda_{p, \beta}\end{array}\right)$, where we use the Dirac matrices in the chiral basis,

$$
\begin{aligned}
& \gamma^{\mu}=\left(\begin{array}{cc}
0 & \sigma^{\mu, \dot{\alpha} \beta} \\
\bar{\sigma}_{\beta \dot{\alpha}}^{\mu} & 0
\end{array}\right)=\left(\begin{array}{cc}
0 & \sqrt{2} \tau^{\mu, \dot{\alpha} \beta} \\
\sqrt{2} \bar{\tau}_{\beta \dot{\alpha}}^{\mu} & 0
\end{array}\right), \\
& \gamma^{5}=i \gamma^{0} \gamma^{1} \gamma^{2} \gamma^{3}=\left(\begin{array}{cc}
-1_{2 \times 2} & 0 \\
0 & 1_{2 \times 2}
\end{array}\right) .
\end{aligned}
$$

Here we have introduced normalized versions of the Pauli matrices ${ }^{4}$ such that, analogous to (2.3), we have

$\operatorname{Tr}\left(\tau^{\mu} \tau^{\nu}\right)=\delta^{\mu \nu} \Leftrightarrow \operatorname{Tr}\left(\tau^{\mu} \bar{\tau}^{\nu}\right)=g^{\mu \nu}$.

The spinors for outgoing fermions and incoming antifermions are then given by

$$
\begin{aligned}
& \bar{u}(p) \equiv u^{\dagger}(p) \gamma^{0}=\left(\left(u_{R}\right)^{\dagger},\left(u_{L}\right)^{\dagger}\right)=\left(\tilde{\lambda}_{p, \dot{\beta}}, \lambda_{p}^{\alpha}\right), \\
& \bar{v}(p) \equiv v(p)^{\dagger} \gamma^{0}=\left(\left(v_{R}\right)^{\dagger},\left(v_{L}\right)^{\dagger}\right)=\left(\tilde{\lambda}_{p, \dot{\beta}}, \lambda_{p}^{\alpha}\right),
\end{aligned}
$$

\footnotetext{
3 Explicit representations of the Weyl spinors are given in Eqs. (A.10) and (A.11).

4 They are also known in the literature as Infeld-van-der-Waerden symbols (see e.g. [62]). Explicit representations of the Pauli matrices are given in (A.2).
} 
such that for example $\bar{u}(p) P_{L}=\left(\left(u_{R}\right)^{\dagger}, 0\right)=\left(\tilde{\lambda}_{p, \dot{\beta}}, 0\right)$ and $\bar{u}(p) P_{R}=\left(0,\left(u_{L}\right)^{\dagger}\right)=\left(0, \lambda_{p}^{\alpha}\right)$, where we have used the Hermitian conjugate relations for massless spinors,

$\left(\lambda_{p, \beta}\right)^{\dagger}=\tilde{\lambda}_{p, \dot{\beta}}$ and $\left(\tilde{\lambda}_{p}^{\dot{\alpha}}\right)^{\dagger}=\lambda_{p}^{\alpha}$,

which implies for the components $\left(\lambda_{p, \beta}\right)^{*}=\left(\tilde{\lambda}_{p, \dot{\beta}}\right)$ and $\left(\tilde{\lambda}_{p}^{\dot{\alpha}}\right)^{*}=\left(\lambda_{p}^{\alpha}\right)$, for $\beta=\dot{\beta}$ and $\dot{\alpha}=\alpha$.

In this paper we will use the convention of counting all particles in a scattering process as outgoing. The four types of outgoing spinors we need to consider are thus

Right-chiral fermion

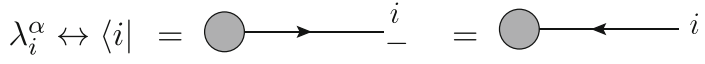

Right-chiral anti-fermion

$\lambda_{j, \alpha} \leftrightarrow|j\rangle=\longleftarrow_{-}^{j}={ }_{-}^{\longrightarrow}{ }^{j}$

Left-chiral fermion

$\tilde{\lambda}_{i, \dot{\alpha}} \leftrightarrow\left[i \mid=\bigcirc \longrightarrow{ }_{+}^{i}=\bigcirc \cdots \cdots \cdots\right.$

Left-chiral anti-fermion

$\left.\tilde{\lambda}_{j}^{\dot{\alpha}} \leftrightarrow \mid j\right]=\bigcirc \longleftarrow \longleftarrow_{+}^{j}=\bigcirc \cdots \cdots$

where we further introduce the common bra-ket notation for Weyl spinors, and where the left graphical rules correspond to the conventional Feynman rules (showing fermion-flow arrow, momentum label and helicity label) whereas in the right pictorial rules, we use dotted lines to denote particles with dotted indices (outgoing positive helicity) and solid lines to denote particles with undotted indices (outgoing negative helicity). ${ }^{5}$ Also note the arrow direction in the rightmost pictorial rules, which - at this point - goes against the fermionflow arrow direction. This arrow direction follows what we dub a chirality flow, i.e. a flow between Weyl spinors in the $(1 / 2,0)$-representation (for dotted lines) or the $(0,1 / 2)$ representation (for solid lines).

We will find situations where the chirality-flow arrow cannot be kept anti-aligned with respect to the fermion-flow arrow. Nevertheless, we adopt the convention of reading all chirality-flow expressions, such as spinor inner products, following the chirality-flow arrow. For convenience we collect our conventions in Tables 1 and 2 in Appendix A.4.

\footnotetext{
${ }^{5}$ For massless, outgoing fermions as well as anti-fermions, the positive and negative helicity states are described by the corresponding left- and right-chiral Weyl spinors respectively, i.e. $\bar{u}^{+}=\left(\left(\bar{u}^{+}\right)_{L},\left(\bar{u}^{+}\right)_{R}\right)=$ $\left(\left(\bar{u}^{+}\right)_{L}, 0\right)$ and $\bar{u}^{-}=\left(\left(\bar{u}^{-}\right)_{L},\left(\bar{u}^{-}\right)_{R}\right)=\left(0,\left(\bar{u}^{-}\right)_{R}\right)$ as well as $v^{+}=$ $\left(\begin{array}{c}\left(v^{+}\right)_{L} \\ \left(v^{+}\right)_{R}\end{array}\right)=\left(\begin{array}{c}\left(v^{+}\right)_{L} \\ 0\end{array}\right)$ and $v^{-}=\left(\begin{array}{c}\left(v^{-}\right)_{L} \\ \left(v^{-}\right)_{R}\end{array}\right)=\left(\begin{array}{c}0 \\ \left(v^{-}\right)_{R}\end{array}\right)$, where we have made the helicity labels explicit.
}

Spinor indices are raised and lowered by the Levi-Civita tensor (sometimes called the spinor metric) $\epsilon^{\alpha \beta} / \epsilon^{\dot{\alpha} \dot{\beta}} / \epsilon_{\alpha \beta} / \epsilon_{\dot{\alpha} \dot{\beta}}$, which we define as

$\epsilon^{12}=-\epsilon^{21}=\epsilon_{21}=-\epsilon_{12}=1$,

such that $\epsilon_{\alpha \beta} \epsilon^{\beta \gamma}=\delta_{\alpha}^{\gamma}$ and $\epsilon^{\dot{\alpha} \dot{\beta}} \epsilon_{\dot{\beta} \dot{\gamma}}=\delta_{\dot{\gamma}}^{\dot{\alpha}}$. With our definition of the $\epsilon$-tensor above, the operations for lowering and raising spinor indices are ${ }^{6}$

$$
\begin{aligned}
\lambda_{p, \alpha} & =\epsilon_{\alpha \beta} \lambda_{p}^{\beta}, \quad \tilde{\lambda}_{p, \dot{\alpha}}=\epsilon_{\dot{\alpha} \dot{\beta}} \tilde{\lambda}_{p}^{\dot{\beta}}, \\
\lambda_{p}^{\alpha} & =\epsilon^{\alpha \beta} \lambda_{p, \beta}, \quad \tilde{\lambda}_{p}^{\dot{\alpha}}=\epsilon^{\dot{\alpha} \dot{\beta}} \tilde{\lambda}_{p, \dot{\beta}} .
\end{aligned}
$$

Considering that $\epsilon$ is the $\operatorname{SL}(2, C)$ invariant object, the definitions for the (antisymmetric, Lorentz invariant) spinor inner products follow as

$$
\begin{aligned}
& \langle i j\rangle \\
& \quad \stackrel{\text { def }}{=}\langle i \| j\rangle=\lambda_{i}^{\alpha} \lambda_{j, \alpha}=\epsilon^{\alpha \beta} \lambda_{i, \beta} \lambda_{j, \alpha}=-\epsilon^{\beta \alpha} \lambda_{i, \beta} \lambda_{j, \alpha} \\
& \quad=-\lambda_{i, \beta} \lambda_{j}^{\beta}=-\langle j i\rangle \\
& {[i j] \stackrel{\text { def }}{=}[i \| j]=\tilde{\lambda}_{i, \dot{\alpha}} \tilde{\lambda}_{j}^{\dot{\alpha}}=\epsilon_{\dot{\alpha}} \tilde{\lambda}_{i}^{\dot{\beta}} \tilde{\lambda}_{j}^{\dot{\alpha}}=-\epsilon_{\dot{\beta} \dot{\alpha}} \tilde{\lambda}_{i}^{\dot{\beta}} \tilde{\lambda}_{j}^{\dot{\alpha}}} \\
& \quad=-\tilde{\lambda}_{i}^{\dot{\beta}} \tilde{\lambda}_{j, \dot{\beta}}=-[j i],
\end{aligned}
$$

implying in particular $\langle i i\rangle=[i i]=0$. Having defined spinors and their graphical representations in the chiralityflow picture, we can represent the spinor inner products pictorially as well,
$\langle i j\rangle=i \longrightarrow j, \quad\langle j i\rangle=i \longleftarrow j$,
$[i j]=i_{\ldots \ldots \ldots} \ldots \ldots, \quad[j i]=i_{\ldots \ldots \ldots} \ldots \ldots$

where we read following the chirality-flow arrow.

Any scattering amplitude can be written in terms of Lorentz-invariant spinor inner products, and in the following sections we will see that we can always find corresponding graphical representations for its Feynman diagrams in terms of chirality-flow lines.

\subsection{Four-vectors}

Next, we consider Lorentz four-vectors. A four-vector $p_{\mu}$ can be mapped to Hermitian $2 \times 2$-matrices, or bispinors, ${ }^{7}$

$p^{\dot{\alpha} \beta} \stackrel{\text { def }}{=} p_{\mu} \tau^{\mu, \dot{\alpha} \beta}=\frac{1}{\sqrt{2}} p_{\mu} \sigma^{\mu, \dot{\alpha} \beta}, \quad \not p \stackrel{\text { def }}{=} p_{\mu} \sigma^{\mu}$

$\bar{p}_{\alpha \dot{\beta}} \stackrel{\text { def }}{=} p_{\mu} \bar{\tau}_{\alpha \dot{\beta}}^{\mu}=\frac{1}{\sqrt{2}} p_{\mu} \bar{\sigma}_{\alpha \dot{\beta}}^{\mu}, \quad \quad \not p \stackrel{\text { def }}{=} p_{\mu} \bar{\sigma}^{\mu}$,

$\overline{{ }^{6} \text { Note that } \lambda_{p, \alpha}}=-\epsilon_{\beta \alpha} \lambda_{p}^{\beta}=-\lambda_{p}^{\beta} \epsilon_{\beta \alpha}$.

7 Explicit representations of momentum bispinors are given in Eqs. (A.25) and (A.26). 
where we have introduced a slash notation for the momentum bispinors, not to be confused with the Feynman slash. The ordinary Lorentz four-vector transformation rules are recovered by boosting and rotating the bispinors as indicated by the spinor index structure, which translates to the statement that Lorentz four-vectors transform under the $(1 / 2,1 / 2)=$ $(1 / 2,0) \otimes(0,1 / 2)$ representation of the (restricted) Lorentz group.

Translating the index structure into the chirality-flow formalism allows us to define a convenient momentum-dot notation

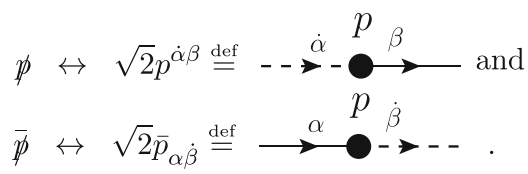

If a momentum $p$ is massless, i.e. light-like, the corresponding momentum bispinors can be expressed as outer products, or dyads, of Weyl spinors,

$\not p=\mid p]\langle p| \leftrightarrow \quad \sqrt{2} p^{\dot{\alpha} \beta}=\tilde{\lambda}_{p}^{\dot{\alpha}} \lambda_{p}^{\beta}, \quad$ for $p^{2}=0$

$\bar{p}=|p\rangle\left[p \mid \leftrightarrow \sqrt{2} \bar{p}_{\alpha \dot{\beta}}=\lambda_{p, \alpha} \tilde{\lambda}_{p, \dot{\beta}}, \quad\right.$ for $p^{2}=0$

or in the chirality-flow picture,

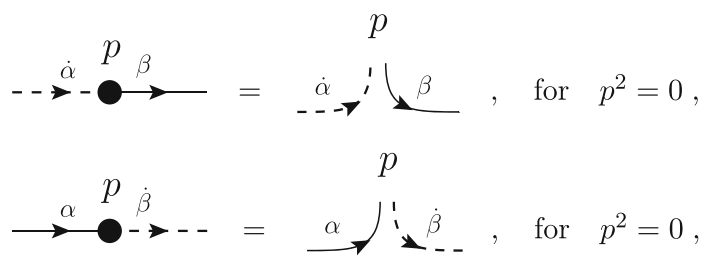

where $p$ in the right graphical rules denotes that the line ends correspond to spinors with momentum $p$. If a momentum $p$ is a linear combination of several external momenta $p_{i}, p=$ $\sum_{i} c_{i} p_{i}$ with $p_{i}^{2}=0-$ which is always the case at tree level - we may use the linearity of Eqs. (3.19) and (3.20) in $p$ to write

$$
\begin{aligned}
& \left.\not p=\sum_{i} c_{i} \mid p_{i}\right]\left\langle p_{i}\right| \leftrightarrow \sqrt{2} p^{\dot{\alpha} \beta}=\sum_{i} c_{i} \tilde{\lambda}_{p_{i}}^{\dot{\alpha}} \lambda_{p_{i}}^{\beta} \\
& \not p=\sum_{i} c_{i}\left|p_{i}\right\rangle\left[p_{i} \mid \leftrightarrow \sqrt{2} \bar{p}_{\alpha \dot{\beta}}=\sum_{i} c_{i} \lambda_{p_{i}, \alpha} \tilde{\lambda}_{p_{i}, \dot{\beta}}\right.
\end{aligned}
$$

or in the chirality-flow picture,

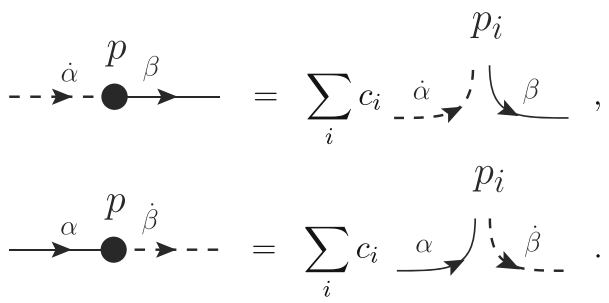

It is sometimes useful to write a four-vector $p^{\mu}$ in terms of a spinor contraction, rather than as a bispinor. We can do so by using (A.4) with (3.16), then using (3.19),

$p^{\mu}=p^{\dot{\alpha} \beta} \bar{\tau}_{\beta \dot{\alpha}}^{\mu}=\frac{1}{\sqrt{2}} \lambda_{p}^{\beta} \bar{\tau}_{\beta \dot{\alpha}}^{\mu} \tilde{\lambda}_{p}^{\dot{\alpha}}=\frac{1}{\sqrt{2}}\left\langle p\left|\bar{\tau}^{\mu}\right| p\right]$,

or similarly, by using (A.4) with (3.17), then using (3.20),

$p^{\mu}=\bar{p}_{\alpha \dot{\beta}} \tau^{\mu, \dot{\beta} \alpha}=\frac{1}{\sqrt{2}} \tilde{\lambda}_{p, \dot{\beta}} \tau^{\mu \dot{\beta} \alpha} \lambda_{p, \alpha}=\frac{1}{\sqrt{2}}\left[p\left|\tau^{\mu}\right| p\right\rangle$.

The above relations will be utilized when proving the chirality-flow picture in Sect. 4, and in the chirality-flow Feynman rules of the fermion propagator and the triple-gluon vertex in Sect. 5.

As a final comment, we remind that for massless spinors the Dirac equation separates into two massless Weyl equations, which in the spinor-helicity formalism take on a particularly simple form. For example, using (3.19) we have

$\left.\left.\not p|p\rangle \stackrel{p^{2}=0}{=}(\mid p]\langle p|\right)|p\rangle=\mid p\right]\langle p p\rangle=0$,

which is easily confirmed to be true, as $\langle p p\rangle=0$ due to the antisymmetry of the spinor inner products (for the other three Weyl equations see Appendix A.3).

\subsection{Polarization vectors}

Aside from external spin-1/2 fermions and momentum fourvectors we also need to treat external vector bosons, for which the outgoing polarization vectors can also be written in terms of Weyl spinors [33,34,40,41,45,46,59],

$\epsilon_{-}^{\mu}\left(p_{i}, r\right)=\frac{\lambda_{i}^{\alpha} \bar{\tau}_{\alpha \dot{\beta}}^{\mu} \tilde{\lambda}_{r}^{\dot{\beta}}}{\tilde{\lambda}_{i, \dot{\gamma}} \tilde{\lambda}_{r}^{\dot{\gamma}}}=\frac{\left\langle i\left|\bar{\tau}^{\mu}\right| r\right]}{[i r]}=\sim_{\sim_{-}^{i}}^{i}$

$\epsilon_{+}^{\mu}\left(p_{i}, r\right)=\frac{\lambda_{r}^{\alpha} \bar{\tau}_{\alpha \dot{\beta}}^{\mu} \tilde{\lambda}_{i}^{\dot{\beta}}}{\lambda_{r}^{\gamma} \lambda_{i, \gamma}}=\frac{\left\langle r\left|\bar{\tau}^{\mu}\right| i\right]}{\langle r i\rangle}={\aleph^{i}}_{i}^{i}$

where $p_{i}$ is the vector boson momentum and $r$ is an arbitrary light-like reference momentum satisfying $p_{i} \cdot r \neq 0$. The subscripts in $\epsilon_{ \pm}$denote helicity labels, and the two polarizations are related by complex conjugation, i.e. $\left(\epsilon_{-}^{\mu}\left(p_{i}, r\right)\right)^{*}=$ $\epsilon_{+}^{\mu}\left(p_{i}, r\right)$.

As for any four-vector, we may trade the Lorentz index in $\epsilon_{ \pm}^{\mu}$ for spinor indices using Eqs. (3.16, 3.17) and (A.7) and 
write them as bispinors,

$\epsilon_{-}^{\dot{\beta} \alpha}\left(p_{i}, r\right)=\frac{\tilde{\lambda}_{r}^{\dot{\beta}} \lambda_{i}^{\alpha}}{\tilde{\lambda}_{i, \dot{\gamma}} \tilde{\lambda}_{r}^{\dot{\gamma}}}=\frac{\mid r]\langle i|}{[i r]}=\frac{1}{[i r]} \bigcirc \cdots-\cdots-$

$\epsilon_{+}^{\dot{\beta} \alpha}\left(p_{i}, r\right)=\frac{\tilde{\lambda}_{i}^{\dot{\beta}} \lambda_{r}^{\alpha}}{\lambda_{r}^{\gamma} \lambda_{i, \gamma}}=\frac{\mid i]\langle r|}{\langle r i\rangle}=\frac{1}{\langle r i\rangle} \bigcirc-\cdots-\cdots-$

From the bispinor representations, we note that an external vector boson has the same numerator structure as an external fermion-antifermion pair, giving a natural graphical interpretation in terms of chirality-flow lines. Also, the two polarization-bispinors are not directly related by complex conjugation. Rather, viewed as matrices we have $\left(\epsilon_{-}\left(p_{i}, r\right)\right)^{*}=\left(\epsilon_{+}\left(p_{i}, r\right)\right)^{\top}$, which follows from the Hermitian conjugation relations between Weyl spinors, (3.5).

As we will see in the next section, we can equally well write the polarization vectors as

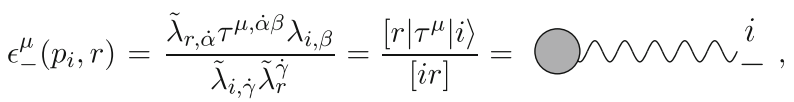

$\epsilon_{+}^{\mu}\left(p_{i}, r\right)=\frac{\tilde{\lambda}_{i, \dot{\alpha}} \tau^{\mu, \dot{\alpha} \beta} \lambda_{r, \beta}}{\lambda_{r}^{\gamma} \lambda_{i, \gamma}}=\frac{\left[i\left|\tau^{\mu}\right| r\right\rangle}{\langle r i\rangle}=\aleph_{\sim_{+}^{i}}^{i}$

\section{Building the chirality-flow picture}

In this section we will see that we can always cast any treelevel Feynman diagram into a linear combination of products of chirality flows, which - when contracted with external spinors - result in spinor inner products. We will also be able to express these chirality flows diagrammatically. This sets the scene for the tree-level chirality-flow Feynman rules in the next section. Here and in the following we work in Feynman gauge.

\subsection{A simple QED example}

Let us first consider the example of single photon exchange between two distinct fermions. We note that in the chiral representation, the Lorentz structure of the fermion-photon vertex may be separated into two parts, corresponding to two different vertices

$$
\begin{aligned}
\bar{u}\left(p_{1}\right) \gamma^{\mu} v\left(p_{2}\right) & =\left(\tilde{\lambda}_{1, \dot{\beta}}, \lambda_{1}^{\alpha}\right)\left(\begin{array}{cc}
0 & \sqrt{2} \tau^{\mu, \dot{\beta} \eta} \\
\sqrt{2} \bar{\tau}_{\alpha \dot{\gamma}}^{\mu} & 0
\end{array}\right)\left(\begin{array}{c}
\tilde{\lambda}_{2}^{\dot{\gamma}} \\
\lambda_{2, \eta}
\end{array}\right) \\
& =\sqrt{2} \tilde{\lambda}_{1, \dot{\beta}} \tau^{\mu, \dot{\beta} \eta} \lambda_{2, \eta}+\sqrt{2} \lambda_{1}^{\alpha} \bar{\tau}_{\alpha \dot{\gamma}}^{\mu} \tilde{\lambda}_{2}^{\dot{\gamma}}
\end{aligned}
$$

For photon exchange, i.e. two fermion-photon vertices with an intermediate photon propagator, there are four possible terms, corresponding to the four possible helicity combinations. Focusing only on the Lorentz structure we have

$$
\begin{aligned}
& \left(\bar{u}\left(p_{1}\right) \gamma^{\mu} v\left(p_{2}\right)\right) g_{\mu \nu}\left(\bar{u}\left(p_{3}\right) \gamma^{\nu} v\left(p_{4}\right)\right) \sim \\
& \left(\tilde{\lambda}_{1, \dot{\alpha}} \tau^{\mu, \dot{\alpha} \beta} \lambda_{2, \beta}\right)\left(\tilde{\lambda}_{3, \dot{\gamma}} \tau_{\mu}^{\dot{\gamma} \eta} \lambda_{4, \eta}\right)+\left(\tilde{\lambda}_{1, \dot{\alpha}} \tau^{\mu, \dot{\alpha} \beta} \lambda_{2, \beta}\right)\left(\lambda_{3}^{\gamma} \bar{\tau}_{\mu, \gamma \dot{\eta}} \tilde{\lambda}_{4}^{\dot{\eta}}\right)+\left(\lambda_{1}^{\alpha} \bar{\tau}_{\alpha \dot{\beta}}^{\mu} \tilde{\lambda}_{2}^{\dot{\beta}}\right)\left(\tilde{\lambda}_{3, \dot{\gamma}} \tau_{\mu}^{\dot{\gamma} \eta} \lambda_{4, \eta}\right)+\left(\lambda_{1}^{\alpha} \bar{\tau}_{\alpha \dot{\beta}}^{\mu} \tilde{\lambda}_{2}^{\dot{\beta}}\right)\left(\lambda_{3}^{\gamma} \bar{\tau}_{\mu, \gamma \dot{\eta}} \tilde{\lambda}_{4}^{\dot{\eta}}\right)
\end{aligned}
$$

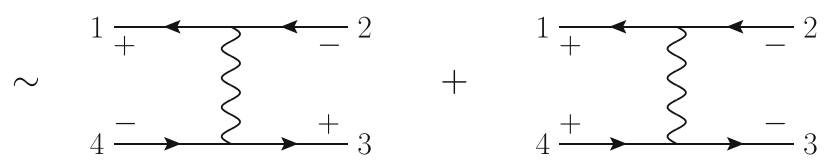
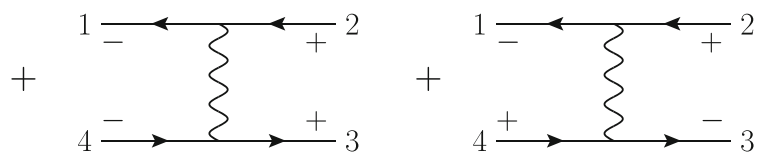

or again as bispinors,

$$
\begin{gathered}
\bar{\epsilon}_{-, \beta \dot{\alpha}}\left(p_{i}, r\right)=\frac{\lambda_{i, \beta} \tilde{\lambda}_{r, \dot{\alpha}}}{\tilde{\lambda}_{i, \dot{\gamma}} \tilde{\lambda}_{r}^{\dot{\gamma}}}=\frac{|i\rangle[r \mid}{[i r]}=\frac{1}{[i r]} \bigcirc \stackrel{-\cdots-{ }^{r}}{i}, \\
\bar{\epsilon}_{+, \beta \dot{\alpha}}\left(p_{i}, r\right)=\frac{\lambda_{r, \beta} \tilde{\lambda}_{i, \dot{\alpha}}}{\lambda_{r}^{\gamma} \lambda_{i, \gamma}}=\frac{|r\rangle[i \mid}{\langle r i\rangle}=\frac{1}{\langle r i\rangle} \bigcirc \stackrel{3.36}{\longrightarrow} r^{i}
\end{gathered}
$$

Some more details are provided at the end of Appendix A.3.
Considering the second or third term in (4.2), having the structure $\tau^{\mu} \bar{\tau}_{\mu}$, we note that the Lorentz indices can be contracted using the Fierz identity. Graphically, for example for the third term, we have in the chirality-flow picture

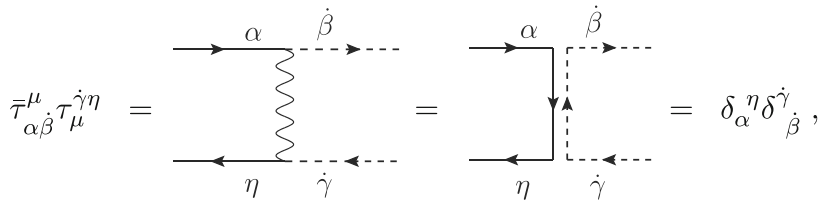


where we have not yet applied the external spinors. This is a graphical embodiment of the Fierz identity, and can be compared to the corresponding identity for SU(3) color, (2.1). The left diagram in (4.3) is a hybrid representation between a conventional Feynman diagram and a diagram in the chiralityflow picture. Here we let the photon line denote the group theory structure only, in analogy with the gluon line in (2.2). ${ }^{8}$ We see that, at least in this case, the Lorentz structure of the photon propagator in the chirality-flow picture may be represented by a double line

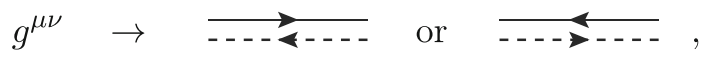

where we defined a double line to be two parallel lines with opposing arrows, where one line is dotted and the other is undotted.

Note that (4.3), when applied to external spinors, results directly in the spinor inner products

$\bar{\tau}_{\alpha \dot{\beta}}^{\mu} \tau_{\mu}^{\dot{\gamma} \eta} \lambda_{1}^{\alpha} \tilde{\lambda}_{2}^{\dot{\beta}} \tilde{\lambda}_{3, \dot{\gamma}} \lambda_{4, \eta}=\langle 14\rangle[32]$,

or pictorially, ${ }^{9}$

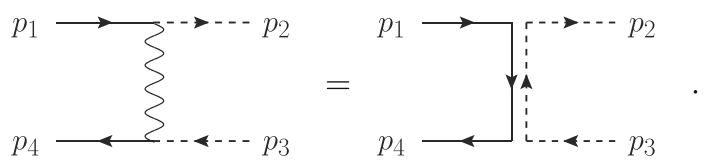

In the first and fourth terms in (4.2), of the forms $\tau^{\mu} \tau_{\mu}$ and $\bar{\tau}^{\mu} \bar{\tau}_{\mu}$ respectively, the arrow directions in the chirality-flow picture would - at this point - not match. For example, for the fourth term we have (using (A.8))

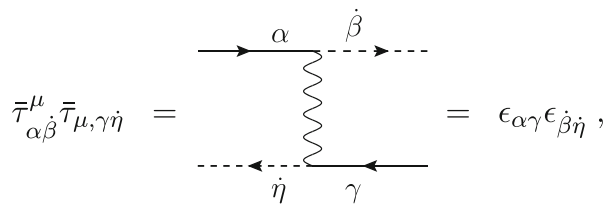

8 The absence of the $1 / N$-suppressed term in (4.3), compared to Eqs. (2.1) or (2.2), can be understood by noting that this term is canceled against the contribution from $\bar{\tau}_{\alpha \dot{\beta}}^{0} \tau_{0}^{\dot{\gamma} \eta}$. An equivalent way of viewing this is that we are summing over the generators of $U(2)$ in (4.3), meaning that we should not expect an additional term.

9 We remark that the graphical appearance of (4.6) is very similar to the one of (4.3), the difference being only in the labels of the external lines. We will usually supply the external lines of a chirality-flow diagram with particle labels, i.e. labels of spinor momenta, but the external lines may in principle also be kept "free", i.e. with spinor indices to act as placeholders, to be supplied with particle labels at some later stage. where we note that the dotted (undotted) lines point away from (towards) each other. Here it is less obvious how to proceed, but we will show that the flow picture as applied in (4.3), the case of matching arrows, can actually be applied here as well.

To see this we use the identity for charge conjugation of a current ${ }^{10}$

$$
\begin{aligned}
& \lambda_{i}^{\alpha} \bar{\tau}_{\alpha \dot{\beta}}^{\mu} \tilde{\lambda}_{j}^{\dot{\beta}} \stackrel{\text { def }}{=} \epsilon^{\alpha \gamma} \epsilon^{\dot{\beta} \dot{\delta}} \lambda_{i, \gamma} \bar{\tau}_{\alpha \dot{\beta}}^{\mu} \tilde{\lambda}_{j, \dot{\delta}} \\
& \quad=\tilde{\lambda}_{j, \dot{\delta}}\left(-\epsilon^{\gamma \alpha}\right)\left(-\epsilon^{\dot{\delta} \dot{\beta}}\right) \bar{\tau}_{\alpha \dot{\beta}}^{\mu} \lambda_{i, \gamma}=\tilde{\lambda}_{j, \dot{\delta}} \tau^{\mu, \dot{\delta} \gamma} \lambda_{i, \gamma},
\end{aligned}
$$

or equivalently

$\left\langle i\left|\bar{\tau}^{\mu}\right| j\right]=\left[j\left|\tau^{\mu}\right| i\right\rangle$,

which we have already used to relate the polarization vectors in Eqs. (3.30) and (3.31) to the ones in Eqs. (3.34) and (3.35) respectively. This means that whenever a $\bar{\tau}$ is squeezed between external spinors we can trade it for a $\tau$, or vice versa, if we also perform the corresponding index lowering or raising operations on the spinors.

The pictorial representation of Eqs. (4.8) and (4.9) in the hybrid representation is

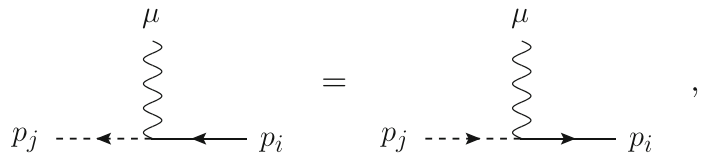

from which we conclude that, for gauge boson exchange between free fermions, the directions of the chirality-flow arrows at a fermion-photon vertex may be flipped such that the resulting diagram is one with matching chiralityflow arrows. Once flipped, the Fierz identity (4.3) may be used such that, for example, the fourth term in (4.2) can be expressed as
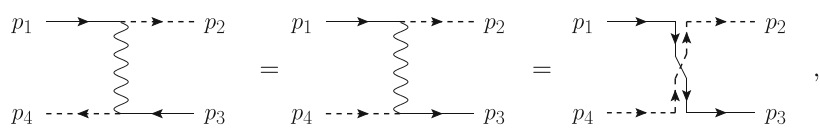

where we have performed the arrow swap at the lower vertex. Note that we could have alternatively performed the arrow swap at the upper vertex. Graphically we see that also in this diagram, the Lorentz structure of the photon propagator in the chirality-flow picture may be represented by a double line with arrows opposing each other, as in (4.4).

Therefore, in the example of single photon exchange between fermions, we see that for every allowed combination of external helicities we can turn the Feynman diagram into a chirality-flow diagram.

\footnotetext{
$\overline{10}$ In the last step we use (A.27), the relation between $\tau$ and $\bar{\tau}$, which can also be seen from the explicit matrix representation for the $\tau$ matrices, (A.2).
} 


\subsection{Proof for QED}

We will now show that this can be done for all QED tree-level Feynman diagrams with explicit external helicities. To do so, we must show that it is always possible to swap the chiralityflow arrows such that we can use the Fierz identity, (4.3), for each contraction of vector indices. While proving this, we will also show that we can always write $g^{\mu \nu}$ (or $g_{\mu \nu}$ ) as a double line with opposing arrows, i.e. that (4.4) always holds.

We have just seen that we can always swap the chiralityflow arrows on a fermion line which emits a single photon, (4.10). Similarly, it is possible to show that we can swap the chirality-flow arrows for a fermion line which emits an arbitrary number of photons, i.e.

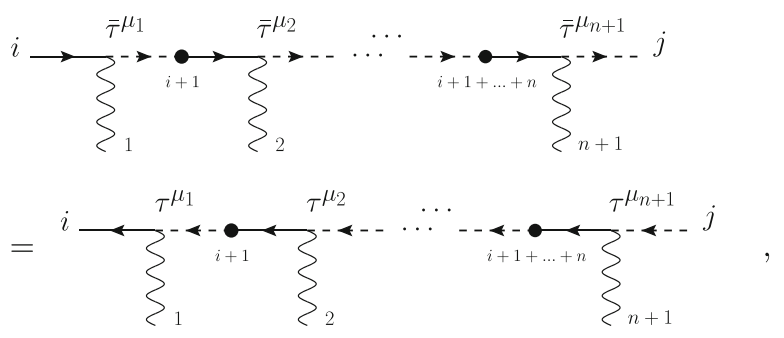

where we use the momentum-dot notation from (3.18) for the fermion propagators. We can prove (4.12) by using Eqs. (3.23-3.26) to write it as

$$
\begin{aligned}
\left.\langle i| \bar{\tau}^{\mu_{1}}(\mid i]\langle i|+| 1]\langle 1|\right) \bar{\tau}^{\mu_{2}} \ldots \bar{\tau}^{\mu_{n}} & \left.(\mid i]\langle i|+| 1]\langle 1|+\cdots+| n]\langle n|) \bar{\tau}^{\mu_{n+1}} \mid j\right] \\
= & {\left[i \mid \tau^{\mu_{1}}\left(|i\rangle[i|+| 1\rangle[1 \mid) \tau^{\mu_{2}} \ldots \tau^{\mu_{n}}\right.\right.} \\
& \left(|i\rangle[i|+| 1\rangle[1|+\cdots+| n\rangle[n \mid) \tau^{\mu_{n+1}}|j\rangle,\right.
\end{aligned}
$$

which is seen by applying (4.9) to transform each $\left\langle k\left|\bar{\tau}^{\mu}\right| l\right] \leftrightarrow$ $\left[l\left|\tau^{\mu}\right| k\right\rangle$.

The photons attached to the fermion line in (4.12) may be either internal or external. If they are external, we use that the Lorentz structure of an external photon is the same as that of a fermion-antifermion pair connected to the fermion line by that photon, i.e. $\epsilon^{\mu} \sim\left\langle i\left|\bar{\tau}^{\mu}\right| j\right]$ as noted at the end of Sect. 3.3. We call such a structure a pseudo-vertex, and for each pseudo-vertex we can always adjust the chirality-flow arrows such that the Fierz identity can be applied.

If a photon in (4.12) is internal, it must be attached to another fermion line. In QED, we can build any Feynman diagram by using internal photons to iteratively stitch together such fermion lines. At tree level, each new fermion line attaches to only one already existing fermion line, so we can freely adjust the chirality-flow arrows on the new line such that the Fierz identity holds at the attachment. Remembering that the external photons are equivalent to fermion lines, we can therefore write any Feynman diagram in such a way that the chirality-flow arrows match, and (4.4) holds. We conclude that any QED tree-level Feynman diagram can be written as a chirality-flow diagram multiplied by scalar factors from vertices, propagators, and external photons.

\subsection{Proof for QCD}

It remains to show that the flow picture can be applied to QCD tree-level Feynman diagrams as well. We begin by remarking that the fermion-boson QCD vertex and the external polarization vectors have the same Lorentz structure as in the QED case. What remains therefore is to understand the Lorentz structures of the triple-gluon vertex, made up of terms $\sim p^{\mu} g^{\nu \rho}$, and the four-gluon vertex, with terms of the form $\sim g^{\mu \nu} g^{\rho \sigma}$.

Let us first ignore factors of $p^{\mu}$ from the triple-gluon vertex. In this case, the metric factors $g^{\mu \nu}$ from the non-abelian vertices (possibly combined with metric factors from propagators) will only act to contract indices from $\tau$ or $\bar{\tau}$ matrices in fermion lines, as in QED. Therefore, we can swap the chirality-flow arrow as required, and (4.4) still holds.

Next, we include the factors of $p^{\mu}$. Using Eqs. (3.27) and (3.28),

$p^{\mu}=\frac{1}{\sqrt{2}}\left\langle p\left|\bar{\tau}^{\mu}\right| p\right]=\frac{1}{\sqrt{2}}\left[p\left|\tau^{\mu}\right| p\right\rangle$,

we see that the momentum $p^{\mu}$ can be viewed as another type of pseudo-vertex, and we can again proceed as in the QED case. We thus conclude that (4.4) still holds, and that any tree-level QCD Feynman diagram with explicit helicities can be cast as a (sum of) chirality-flow diagram(s) multiplied by (Lorentz) scalar factors from vertices, propagators, and external gluons.

\subsection{QCD remarks}

A novel feature of the QCD non-abelian vertices is the existence of disconnected Lorentz structures. For example, in the four-gluon vertex consisting of terms of the form $g^{\mu \nu} g^{\rho \sigma}$, the flow of Lorentz indices in $g^{\mu \nu}$ does not affect the flow of the Lorentz indices in $g^{\rho \sigma}$. This leads to disconnected pieces of a chirality-flow diagram, i.e. spinor lines not related to each other by either momentum dots or a shared double line. We can flip the arrow direction of one disconnected piece without affecting the arrow direction of the other.

Additionally, a new chirality-flow rule for $p^{\mu}$ in the triplegluon vertex must be found. If $p^{\mu}$ is contracted with a fermion line or the polarization vector of an external gluon, it will result in $p^{\mu} \tau_{\mu}=\not p / \sqrt{2}$ (or $p^{\mu} \bar{\tau}_{\mu}=\bar{p} / \sqrt{2}$ ), allowing to use 
(3.18) to identify

$p^{\mu} \rightarrow \frac{1}{\sqrt{2}} \stackrel{p}{\longrightarrow} \longrightarrow \quad$ or $\quad p^{\mu} \rightarrow \frac{1}{\sqrt{2}} \longrightarrow \stackrel{p}{\longrightarrow} \rightarrow-\cdots$,

in these cases.

The remaining situation to consider is when the momentum $p_{i}^{\mu}$ is instead contracted with another momentum $p_{j}^{v}$. In this case, we obtain

$p_{i} \cdot p_{j}=p_{i}^{\mu} p_{j}^{\nu} \operatorname{Tr}\left(\tau_{\mu} \bar{\tau}_{\nu}\right)=\frac{1}{2} \operatorname{Tr}\left(\not p_{i} \bar{p}_{j}\right)=\frac{1}{2} \overbrace{p_{j}}^{p_{i}^{\prime}}$

for which we can choose the chirality-flow arrow independently from the rest of the diagram. Such a term is therefore another example of a disconnected piece of a chirality-flow diagram.

We thus conclude that the chirality-flow rule for $p^{\mu}$ in the triple-gluon vertex can be taken to be (4.15). Note that due to (4.14), we may always use the version of (4.15) required to obtain chirality-flow arrows which match the rest of the diagram.

\section{Chirality-flow Feynman rules}

In this section we will collect the chirality-flow Feynman rules for massless QED and QCD, using the result of the previous section that any tree-level Feynman diagram can be cast as a (sum of) chirality-flow diagram(s). The corresponding rules for external spinors and polarization vectors have been collected in Sects. 3.1 and 3.3 already. For convenience we also collect the full set of Feynman rules, in various representations, in the 'Rosetta stones' in Tables 1 and 2 in Appendix A.4.

\subsection{Vertices}

We start with the fermion-photon vertex. Suppressing spinor indices, it is given by

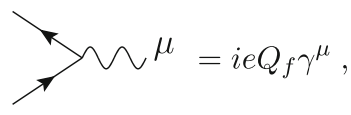

with $e$ and $Q_{f}$ being the electromagnetic coupling constant and the charge respectively. In the Weyl representation we can separate the vertex into two parts,

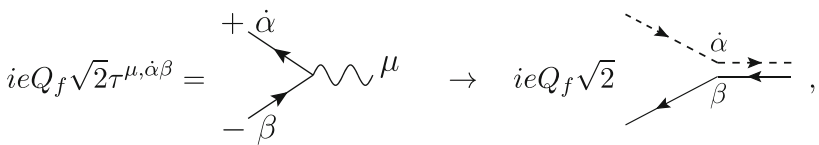

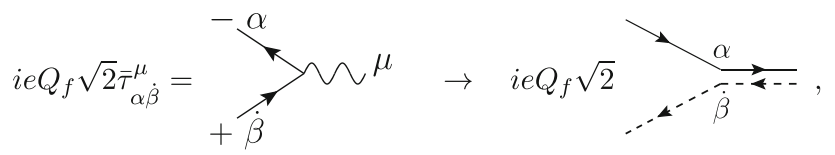

depending on the helicity configurations of the fermions. Here we make the Weyl spinor indices explicit, and the right graphical rules represent the two parts in the chirality-flow picture. If the fermion-antifermion pair is a quark-antiquark pair we have to multiply by an additional $\delta_{i \bar{j}}$, with $i$ and $\bar{J}$ being the color indices of the (outgoing) quark and antiquark respectively.

The quark-gluon vertex is similarly given by ${ }^{11}$

$\gamma_{\bar{\jmath}}^{i} \infty \infty \times \infty, \mu=i \frac{g_{s}}{\sqrt{2}} t_{i \bar{\jmath}}^{a} \gamma^{\mu}$

with $g_{s}$ denoting the strong coupling constant. Note that, comparing to the (perhaps most common) convention where $\operatorname{Tr}\left(t^{a} t^{b}\right)=\frac{1}{2} \delta^{a b}$, our generators and structure constants are a factor $\sqrt{2}$ larger, which affects the normalization of our $\mathrm{QCD}$ vertices.

In the Weyl representation we can again separate the vertex into two parts,

$i g_{s} t_{i \bar{\jmath}}^{a} \tau^{\mu, \dot{\alpha} \beta}=\sum_{-\bar{\jmath}, \beta}^{+i, \dot{\alpha}} \infty 000 a, \mu \rightarrow i g_{s} t_{i \bar{\jmath}}^{a}$

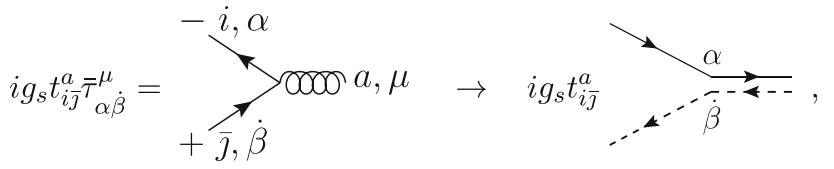

depending on the helicity configurations of the quarks.

We also need the three- and four-gluon vertices. For the three-gluon vertex, the color and kinematic parts factorize trivially,

$\overline{11}$ Note that $i$ and $\bar{j}$ are the color indices of outgoing quarks and antiquarks respectively, meaning that indices on $t_{i \bar{j}}^{a}$, as well as colorflow arrows are read against the fermion flow. Correspondingly, the reading direction of $f^{a b c}$ is clockwise. Opposite reading directions, for both quark color (along fermion arrow) and structure constants (counter clockwise) may be imposed without introducing additional signs. In the color-flow picture, and with our conventions in Eqs. (2.1) and (2.3), $t_{i \bar{j}}^{a}$ is translated to $\delta_{i \bar{i}_{a}} \delta_{i_{a} \bar{\jmath}}$. 


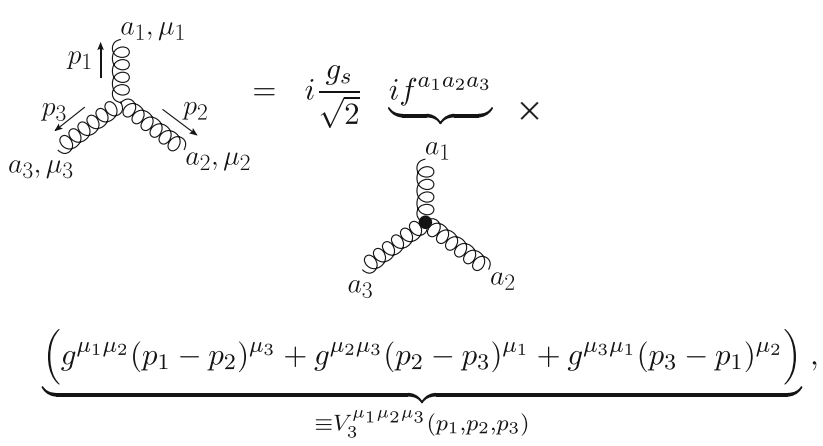

where we take the graph with only color indices and a dot in the vertex to equal if $f^{a_{1} a_{2} a_{3}}$. The kinematic part can also be written in a more condensed form as $V_{3}^{\mu_{1} \mu_{2} \mu_{3}}\left(p_{1}, p_{2}, p_{3}\right)=$ $\sum_{Z(1,2,3)} g^{\mu_{1} \mu_{2}}\left(p_{1}-p_{2}\right)^{\mu_{3}}$, where $Z(1,2,3)$ denotes the set of cyclic permutations of the integers 1, 2, 3. Using Eqs. (4.4) and (4.15) to translate $g^{\mu_{1} \mu_{2}}$ and $\left(p_{1}-p_{2}\right)^{\mu_{3}}$ etc. to the chirality-flow picture, we get
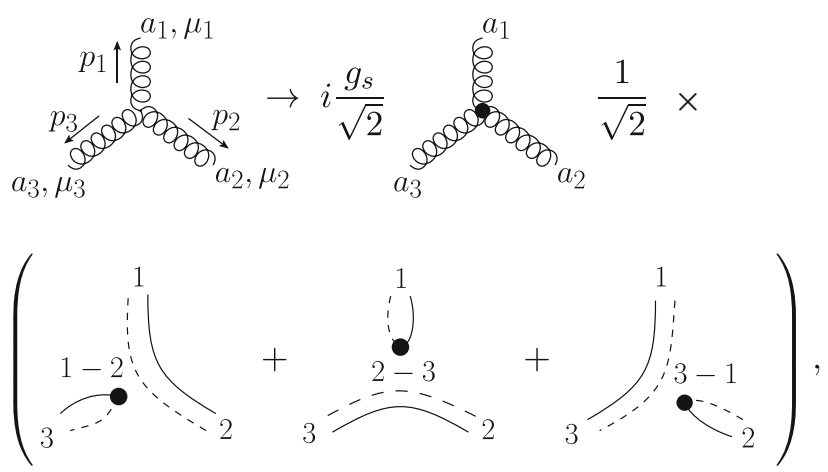

where the chirality-flow arrows have been removed since they must flow in different directions for different terms in the sum. However, the arrow directions of the dotted and undotted chirality-flow lines must always oppose each other in the double lines (from the metric), and form a continuous flow in the lines joined by a momentum-dot (from the momentum). Note that since we only consider tree-level diagrams with massless particles, the momentum parts can always be written as linear combinations of external momenta $p_{i}$, with $p_{i}^{2}=$ 0, such that we can use Eqs. (3.23-3.26) to reduce the corresponding momentum bispinors to outer products, or dyads, of momentum spinors. Using Eqs. (2.4) and (2.1), and realizing that $V_{3}^{\mu_{1} \mu_{2} \mu_{3}}\left(p_{1}, p_{2}, p_{3}\right)=-V_{3}^{\mu_{1} \mu_{3} \mu_{2}}\left(p_{1}, p_{3}, p_{2}\right)$, we can further translate the color part of the three-gluon vertex to the color-flow picture, such that
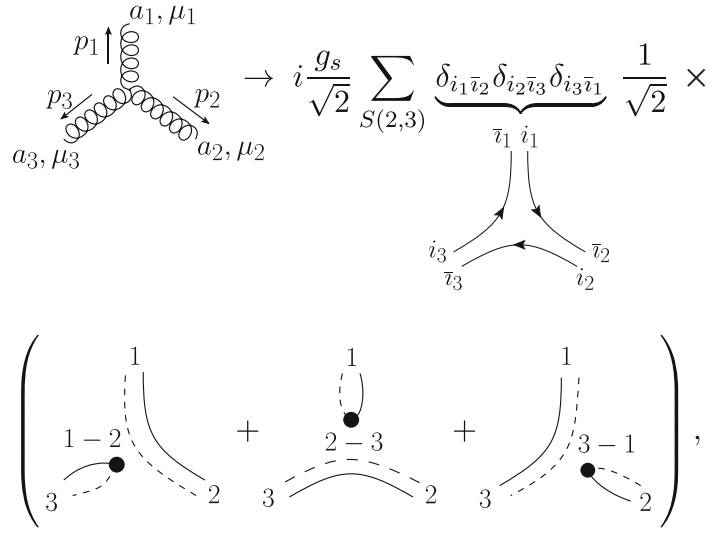

where $S(2,3)$ denotes the set of permutations of the integers 2, 3, and where the lines below the Kronecker deltas represent the color flows. ${ }^{12}$

For the four-gluon vertex, we translate the metric to the chirality-flow picture in the same way as before,
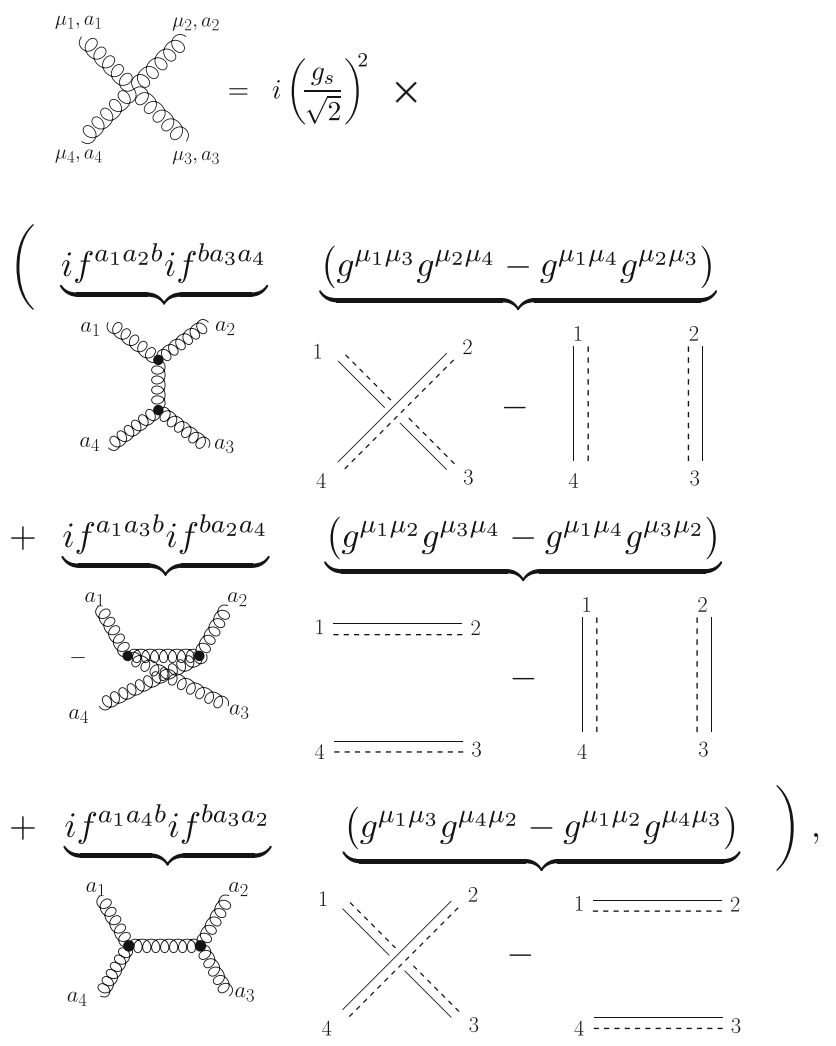

where we also give the conventional graphical representation for the color factors, as well as the graphical chirality-flow representation for the kinematic parts. Again, the arrow directions in the chirality-flow lines must oppose each other and be adjusted to match the rest of the diagram.

The four-gluon vertex can also be written in a more condensed form,

12 In the trace basis, and with our conventions in Eqs. (2.1) and (2.3, 2.4), this vertex is $i g_{s} / \sqrt{2} \sum_{S(2,3)} \operatorname{Tr}\left(t^{a_{1}} t^{a_{2}} t^{a_{3}}\right) V_{3}^{\mu_{1} \mu_{2} \mu_{3}}\left(p_{1}, p_{2}, p_{3}\right)$. 


$$
\begin{aligned}
& C_{\mu_{4}, a_{4}}^{\mu_{1}, a_{1}} \sigma_{\sigma_{\mu_{3}, a_{3}}^{\mu_{2}, a_{2}}}=i\left(\frac{g_{s}}{\sqrt{2}}\right)^{2} \times \\
& \sum_{z(2,3,4)} i f^{a_{1} a_{2} b} i f^{b a_{3} a_{4}}\left(g^{\mu_{1} \mu_{3}} g^{\mu_{2} \mu_{4}}-g^{\mu_{1} \mu_{4}} g^{\mu_{2} \mu_{3}}\right),
\end{aligned}
$$

or if we sort by the same metric factors,

$$
\begin{aligned}
& C_{\mu_{4}, a_{4}}^{\mu_{1}, a_{1}} \sigma_{\sigma_{\mu_{3}, a_{3}}^{\mu_{2}, a_{2}}}=i\left(\frac{g_{s}}{\sqrt{2}}\right)^{2} \times \\
& \sum_{Z(2,3,4)} g^{\mu_{1} \mu_{3}} g^{\mu_{4} \mu_{2}}\left(i f^{a_{1} a_{2} b} i f^{b a_{3} a_{4}}+i f^{a_{1} a_{4} b} i f^{b a_{3} a_{2}}\right) .
\end{aligned}
$$

Translating to the chirality-flow picture, using the conventional graphical representation for the color structure, we note that this corresponds to sorting by chirality flows, such that $\underset{\mu_{4}, a_{4}}{\log _{2}^{\mu_{2}, a_{1}}} \rightarrow i\left(\frac{g_{s}}{\sqrt{2}}\right)^{2} \times$

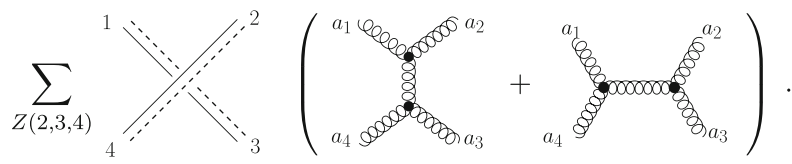

Note that the gluons which are chirality-flow connected are not connected in the structure constants. Finally, we can use Eqs. (2.1) and (2.4) to translate the color parts of the four-gluon vertex to the color-flow picture, such that

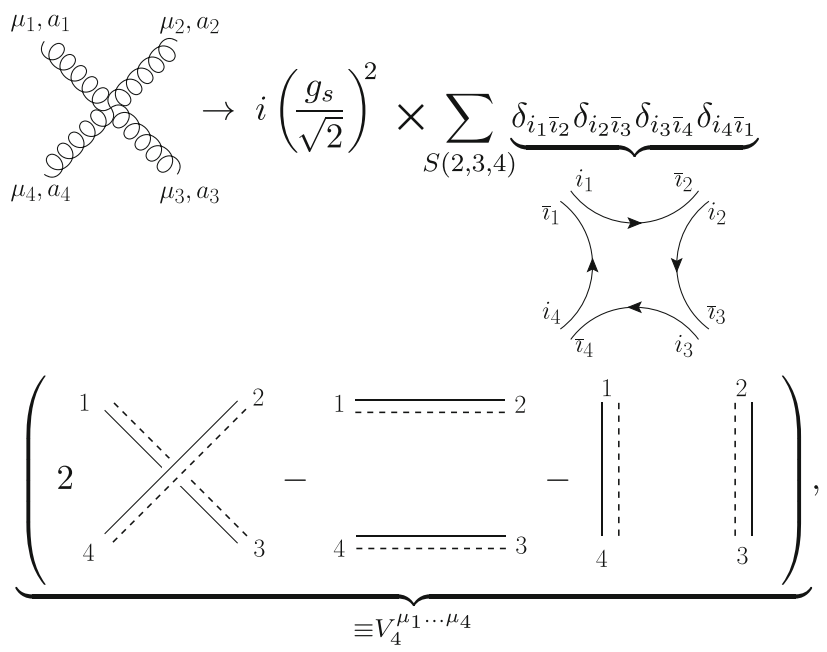

where the lines below the Kronecker deltas represent the color flows. ${ }^{13}$ The lines in the graphs in parentheses represent chirality flows, and we note that they correspond to the Lorentz structure of the kinematic part $V_{4}^{\mu_{1} \ldots \mu_{4}}=$ $\left(2 g^{\mu_{1} \mu_{3}} g^{\mu_{4} \mu_{2}}-g^{\mu_{1} \mu_{2}} g^{\mu_{3} \mu_{4}}-g^{\mu_{1} \mu_{4}} g^{\mu_{2} \mu_{3}}\right)$ in the color-flow decomposition.

\subsection{Propagators}

We also need the propagators for fermions and vector bosons. Starting with the propagator for a massless fermion with momentum $p$ in QED

$$
\stackrel{\stackrel{p}{\longleftarrow}}{\longleftarrow}=\frac{i p_{\mu} \gamma^{\mu}}{p^{2}}=\frac{i}{p^{2}} \sqrt{2}\left(\begin{array}{cc}
0 & p_{\mu} \tau^{\mu} \\
p_{\mu} \bar{\tau}^{\mu} & 0
\end{array}\right)=\frac{i}{p^{2}}\left(\begin{array}{cc}
0 & \not p \\
\not p & 0
\end{array}\right),
$$

we see that - like the fermion-photon vertex - the fermion propagator separates into two parts,

$$
\begin{aligned}
& \frac{i}{p^{2}} \not p \rightarrow \frac{i}{p^{2}} \sqrt{2} p^{\dot{\alpha} \beta}=\frac{i}{p^{2}} \stackrel{\dot{\alpha}}{p} \stackrel{p}{\beta} \stackrel{\bigcirc}{\longrightarrow},
\end{aligned}
$$

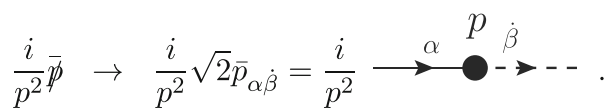

Note that since we only consider tree-level diagrams with massless particles, the momentum can always be written as a linear combination of external momenta $p_{i}$, with $p_{i}^{2}=0$, such that we can use Eqs. (3.23-3.26) to reduce the corresponding momentum bispinors to outer products, or dyads, of momentum spinors. In the case that the fermion is a quark we have to multiply by an additional $\delta_{i \bar{j}}$, with $i$ and $\bar{j}$ being the color indices of the two ends of the quark propagator.

The propagator for a photon with momentum $p$ is given by

$\mu \stackrel{p}{\sim} \sim \nu=-i \frac{g_{\mu \nu}}{p^{2}}$.

We recall from the previous section that - as for the threeand four-gluon vertices - considering a full diagram, we can translate $g^{\mu \nu}$ to the chirality-flow picture using (4.4), such that

$$
\begin{aligned}
& -i \frac{g_{\mu \nu}}{p^{2}}=\mu \rightsquigarrow \stackrel{p}{\sim} \sim^{\nu} \\
& \rightarrow \quad-\frac{i}{p^{2}} \underset{\cdots \cdots-1}{\longrightarrow} \text { or } \quad-\frac{i}{p^{2}} \stackrel{-\cdots}{\longleftarrow},
\end{aligned}
$$

for which the arrow directions of the dotted and undotted lines in the double line must oppose each other, but should be adjusted to match the rest of the diagram. In the case of

\footnotetext{
13 In the trace basis, and with our conventions in Eqs. (2.1) and (2.3, 2.4), this vertex is $i g_{s}^{2} / 2 \sum_{S(2,3,4)} \operatorname{Tr}\left(t^{a_{1}} t^{a_{2}} t^{a_{3}} t^{a_{4}}\right) V_{4}^{\mu_{1} \mu_{2} \mu_{3} \mu_{4}}$.
} 
a gluon propagator we simply multiply by an additional $\delta^{a b}$, with $a$ and $b$ being the color indices of the two ends of the gluon propagator. ${ }^{14}$

To conclude this section, we note that we have formulated a set of chirality-flow rules, analogous to color-flow rules. In fact, in a sense, the flow picture works even better here than in the case of color, since for color we have to bear in mind the $1 / N$ suppressed term in (2.1) for the gluon propagator, as well as for external gluons upon squaring - as long as we are not considering purely gluonic processes. For the Lorentz structure, this complication does not arise.

\subsection{Application}

In the following we give a "recipe" for using the chiralityflow Feynman rules. The Feynman rules are conveniently collected in Tables 1 and 2, and concrete examples are given in Sect. 6.

Consider a Feynman diagram with a given combination of external (outgoing) helicities:

1. Collect all factors of $\pm i, \sqrt{2}$ and coupling constants from vertices (see Sect. 5.1), as well as denominators from propagators (Sect. 5.2) and external polarization vectors (Sect. 3.3).

2. Assign chirality-flow lines, i.e. dotted and undotted lines. Ignore chirality-flow directions in this step. When assigning internal momentum labels to momentum-dots, write the corresponding momentum in terms of external momenta, directed as usually in Feynman diagrams.

- External fermions with positive or negative helicity are assigned a single dotted or undotted line, respectively, and a momentum label (see Eqs. (3.6-3.9)).

- External vector bosons are assigned a double line and two momentum labels; the line corresponding to the physical helicity (the dotted line for positive helicity, the undotted line for negative helicity) is assigned the physical momentum whereas the other line is assigned the reference momentum (see Eqs. (3.32, 3.33 ) and $(3.36,3.37))$.

- Vector boson propagators are assigned a double line, one dotted and one undotted (see (5.19)).

- Fermion propagators are assigned a pair of successive lines, turning from dotted to undotted (or vice versa), joined by a momentum-dot, with the corresponding momentum label (see Eqs. (5.16, 5.17)).

- Using the appropriate vertices, all lines are connected in the only possible way for the Feynman diagram

14 In the color-flow picture, and with our conventions in Eqs. (2.1) and (2.3), $\delta^{a b}$ is translated to $\delta_{i_{a} \bar{l}_{b}} \delta_{i_{b} \bar{l}_{a}}-1 / N \delta_{i_{a} \bar{l}_{a}} \delta_{i_{b} \bar{l}_{b}}$. to form a chirality-flow diagram. For photon- and gluon-fermion vertices, use either one of the chiralityflow structures in (5.2) or (5.3) (equivalently (5.5) or (5.6)), for three-gluon vertices use the sum of chirality-flow structures in (5.8) (or (5.9) for the color-ordered approach) and for four-gluon vertices use the chirality-flow structures in (5.13) (or (5.14) for the color-ordered approach).

3. Assign chirality-flow directions. Start with any external chirality-flow line and assign to it a chirality-flow arrow in an arbitrary direction. ${ }^{15}$ Follow the line through the chirality-flow diagram, continuing through any potential momentum-dot, and assign chirality-flow arrows in the same direction. Assign the other arrow directions such that double lines from gauge bosons have opposing arrows. Note that non-abelian vertices will give rise to disconnected pieces (see Sect. 4.4). For each such disconnected piece, independently apply the above arrow direction rules.

Due to the sums of chirality flows from the non-abelian vertices, each Feynman diagram is now turned into a sum of chirality-flow diagrams. Multiplied by the collected factors in step 1 , as well as by potential color factors, ${ }^{16}$ we obtain the result of the Feynman diagram without any non-trivial algebraic manipulation.

If it is desired to obtain the result in conventional form with spinor brackets, expand the momentum-dots and translate the lines to spinor inner products.

\section{Examples}

In order to demonstrate how the chirality-flow picture is applied, we give some examples. We remind the reader that we adopt the convention of counting all particles in a scattering process as outgoing. ${ }^{17}$ The recipe for turning a Feynman diagram into a chirality-flow diagram is given in Sect. 5.3, and for convenience the Feynman and chirality-flow rules are collected in Tables 1 and 2 .

15 In a sum of Feynman diagrams there is no rule where and how to set the initial chirality-flow arrow. As shown in the previous section, chirality-flow arrow swaps are identity operations, and performing them for each Feynman diagram independently can therefore not introduce any relative minus signs.

16 Note that in the case of four-gluon vertices the color parts do not trivially factorize. Due to this, depending on whether or not we work in a color-ordered approach, color factors may or may not be collected globally in front of the Feynman diagram; i.e. different chirality flows may have different color factors.

17 We remind the reader that after crossing from incoming to outgoing states, a (left-chiral) negative helicity incoming particle for instance turns into a (left-chiral) positive helicity outgoing anti-particle, etc. 
$6.1 e^{+} e^{-} \rightarrow \mu^{+} \mu^{-}$

We begin with a simple example of electron-positron annihilation, creating a muon-antimuon pair through photon exchange. We follow the recipe in Sect. 5.3 and arbitrarily choose one of the possible helicity configurations for the external fermions, considering $M\left(0 \rightarrow e_{L}^{-} e_{R}^{+} \mu_{L}^{-} \mu_{R}^{+}\right)$.

Step 1 asks to collect prefactors and denominators, while step 2 requires assigning dotted lines to positive helicity particles and undotted lines to negative helicity particles, giving

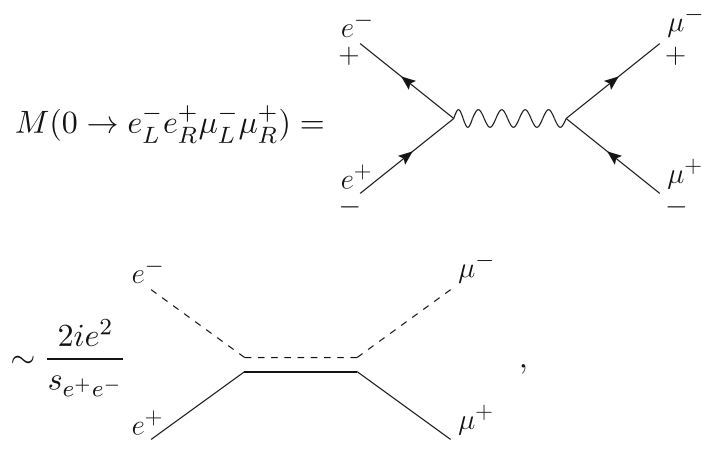

with $s_{e^{+} e^{-}}=\left(p_{e^{+}}+p_{e^{-}}\right)^{2}=2 p_{e^{+}} \cdot p_{e^{-}}$. From the chiralityflow diagram, we see that we no longer need the helicity labels, since the dotted and undotted lines give the same information. Step 3 advises us how to add chirality-flow arrows. We arbitrarily choose the chirality-flow arrow from the $e^{-}$ to point inward and follow its line through the chirality-flow diagram to the $\mu^{-}$, which then has its chirality-flow arrow pointing outward. The arrow on the solid line is then fixed by the double line from the photon propagator; it has to be opposite to that of the dotted line, such that

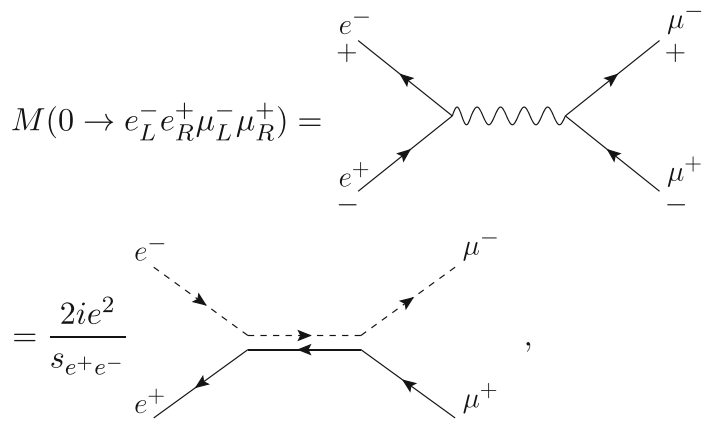

which is our final result. The directed dotted and solid lines are equivalent to the spinor inner products which we may, if desired, convert to the more familiar square and angled brackets using Eqs. (3.14) and (3.15) to obtain $M(0 \rightarrow$ $\left.e_{L}^{-} e_{R}^{+} \mu_{L}^{-} \mu_{R}^{+}\right)=\frac{2 i e^{2}}{s_{e^{+} e^{-}}}\left[e^{-} \mu^{-}\right]\left\langle\mu^{+} e^{+}\right\rangle$.
The only other non-trivial diagram needed for calculating the helicity-summed result is generated by swapping the helicities of the muons,
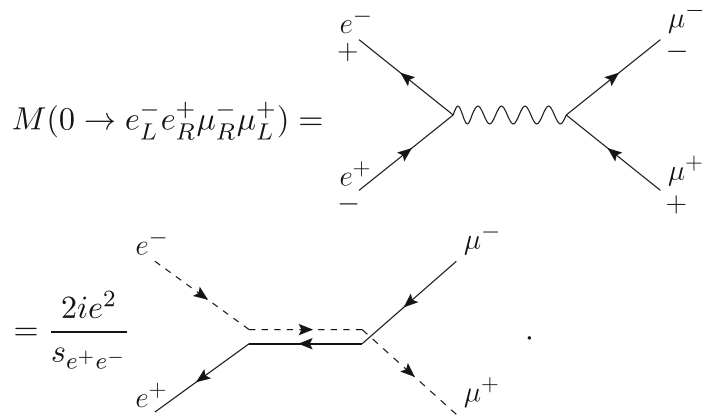

Using Eqs. (3.14) and (3.15), we get $M\left(0 \rightarrow e_{L}^{-} e_{R}^{+} \mu_{R}^{-} \mu_{L}^{+}\right)$ $=\frac{2 i e^{2}}{s_{e^{+} e^{-}}}\left[e^{-} \mu^{+}\right]\left\langle\mu^{-} e^{+}\right\rangle$. The remaining two helicity configurations needed for calculating the helicity-summed result are simply given by exchanging the dotted and solid lines in the chirality-flow diagrams in Eqs. (6.2) and (6.3).

While the above is a simple example - also in the ordinary spinor-helicity formalism, we stress that we did not need to perform a single algebraic manipulation to arrive at the result.

$6.2 e^{+} e^{-} \rightarrow \mu^{+} \mu^{-} \gamma$

As our next example we consider the same process, but with an additional photon radiated externally. This introduces a fermion propagator and a polarization vector. We choose a particular helicity configuration to start with, considering the amplitude $M\left(0 \rightarrow e_{L}^{-} e_{R}^{+} \mu_{L}^{-} \mu_{R}^{+} \gamma_{1}^{+}\right)$. One diagram for this process has the photon emitted from the $\mu^{-}$. Steps 1 and 2 from the recipe ask to draw the chirality-flow structure, giving
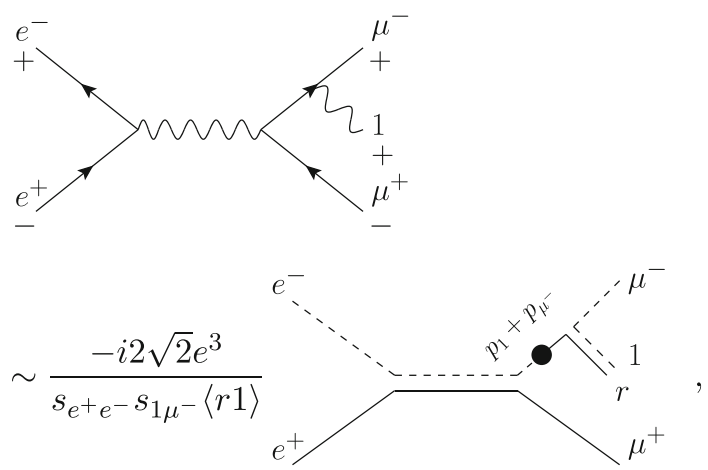

where $r$ denotes the reference momentum of the external photon, the fermion propagator has momentum $p_{1}+p_{\mu^{-}}$and we have used the shorthand notation $p_{1} \rightarrow 1$ to denote the photon momentum. Step 3 is to assign chirality-flow arrows. Following the same procedure as in the previous example, remembering to continue the flow through the momentumdot, we get 

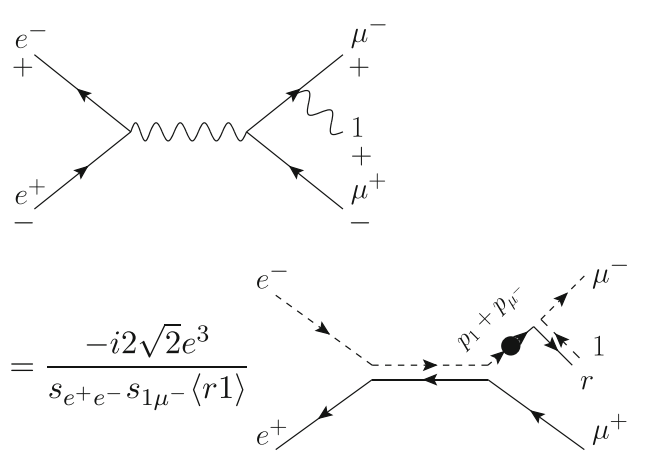

This is in principle our final result, as it contains all the spinor inner products in the diagram. To write out the inner products as square or angled brackets, we first expand the momentumdot using (3.25), and then use Eqs. (3.14) and (3.15) to obtain

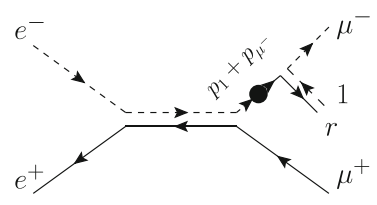

$=\left(\left[e^{-} 1\right]\langle 1 r\rangle+\left[e^{-} \mu^{-}\right]\left\langle\mu^{-} r\right\rangle\right)\left[1 \mu^{-}\right]\left\langle\mu^{+} e^{+}\right\rangle$.
The diagram with the photon emitted from the $\mu^{+}$has a similar structure,
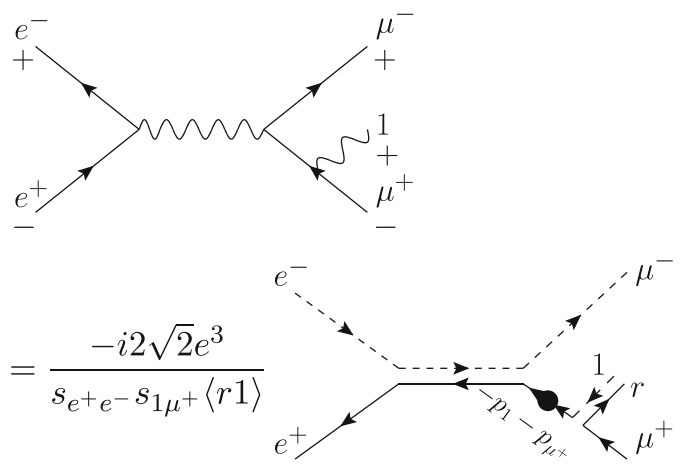

$=\frac{-i 2 \sqrt{2} e^{3}}{s_{e^{+} e^{-}} s_{1 \mu^{+}}\langle r 1\rangle}\left[e^{-} \mu^{-}\right]\left\langle\mu^{+} r\right\rangle\left(-0-\left[1 \mu^{+}\right]\left\langle\mu^{+} e^{+}\right\rangle\right)$,

where in the last step we have used that [11] $=0$.

Following the same procedure to obtain results for the remaining two diagrams gives

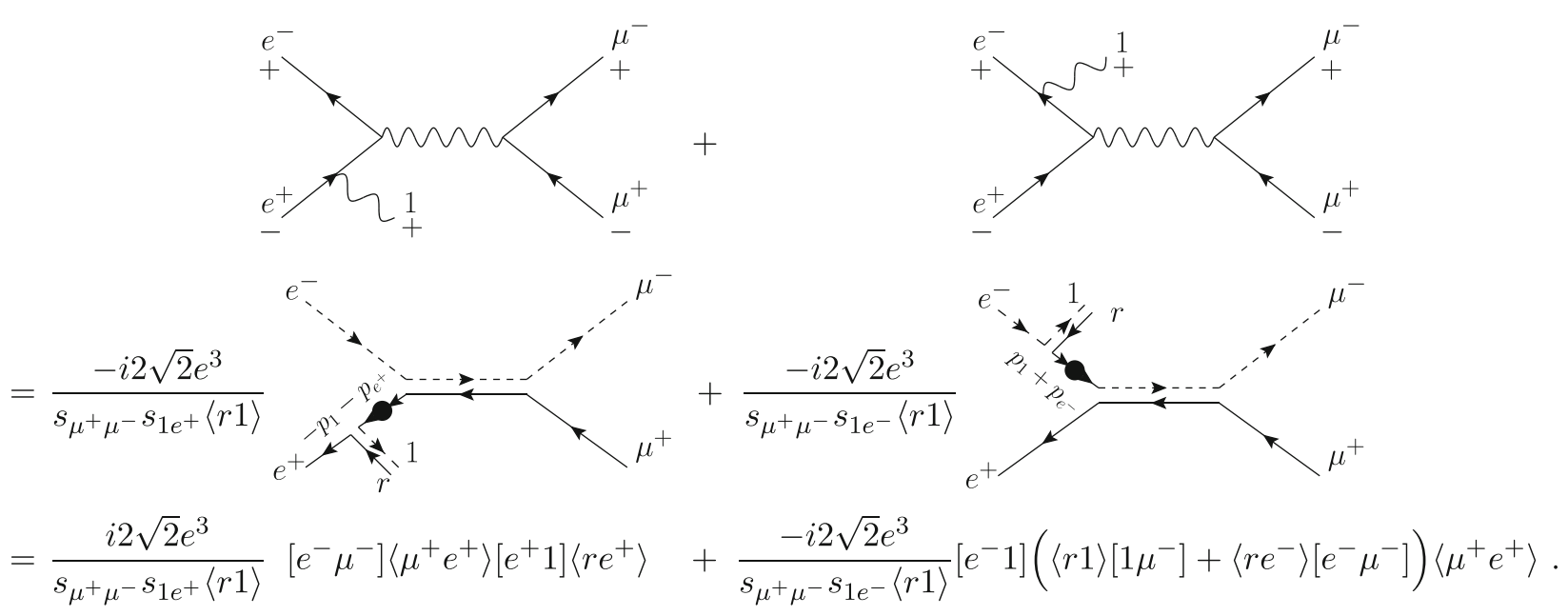

Note that in the results above, we have left the reference momentum of the external photon unassigned. We may simplify the results by choosing it appropriately, ${ }^{18}$ to generate spinor inner products of the form $\langle i i\rangle=[i i]=0$. We stress again that we did not need to perform a single algebraic manipulation, other than to expand momentum-dots, to arrive at the results.

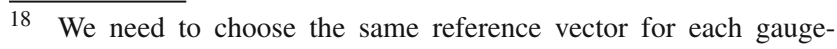
invariant subset of diagrams. 


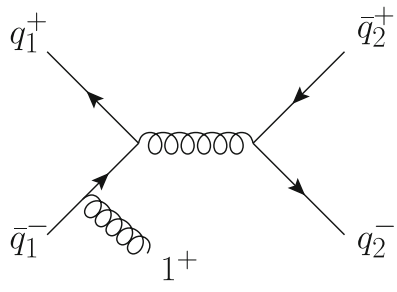

(a)

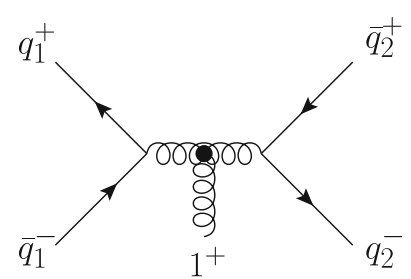

(b)

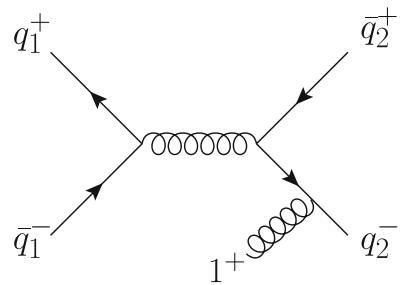

(c)

Fig. 1 The three diagrams contributing to the partial helicity amplitude $M\left(0 \rightarrow q_{2}^{-} 1^{+} \bar{q}_{1}^{-} q_{1}^{+} \bar{q}_{2}^{+}\right)$multiplying the color factor $t_{q_{2}}^{a_{1}} \bar{q}_{1} \delta_{q_{1}} \bar{q}_{2}$

We also remark on the simplicity of the result: in QED every Feynman diagram gives a single chirality-flow graph, where every spinor line is contracted with the "nearest" (closest possible, following the chirality flow) spinor of the same kind.

\section{$6.3 q_{1} \bar{q}_{1} \rightarrow q_{2} \bar{q}_{2} g$}

We now consider our first QCD example, $q_{1} \bar{q}_{1} \rightarrow q_{2} \bar{q}_{2} g$. For this example we will - for comparison - also go through the standard spinor-helicity calculation. We will also for the first time encounter a disconnected chirality-flow structure.

There are six Feynman diagrams in total, and the color structure can be decomposed into four linearly independent basis vectors (color factors). We will consider the (colorordered) partial helicity amplitude $M\left(0 \rightarrow q_{2}^{-} 1^{+} \bar{q}_{1}^{-} q_{1}^{+} \bar{q}_{2}^{+}\right)$ multiplying the color factor $t_{q_{2} \bar{q}_{1}}^{a_{1}} \delta_{q_{1} \bar{q}_{2}}$, which has contributions from the three diagrams in Fig. 1.

Using the standard spinor-helicity method, the Lorentz structure of the diagram in Fig. 1a is given by

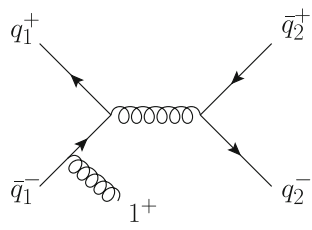

$\rightarrow \frac{-i g_{s}^{3}}{s_{1 \bar{q}_{1}} s_{q_{2} \bar{q}_{2}}}\left[q_{1}\left|\tau^{\mu}\right|-\left(\overline{\not p}_{1}+\overline{\not p}_{\bar{q}_{1}}\right)\left|\epsilon_{1}^{+}\right| \bar{q}_{1}\right\rangle\left\langle q_{2}\left|\bar{\tau}_{\mu}\right| \bar{q}_{2}\right]$

$=\frac{-i g_{s}^{3}}{s_{1 \bar{q}_{1}} s_{q_{2} \bar{q}_{2}}\langle r 1\rangle}\left(-\left[q_{1}\left|\tau^{\mu}\right| 1\right\rangle[11]\left\langle r \bar{q}_{1}\right\rangle\right.$

$$
\left.-\left[q_{1}\left|\tau^{\mu}\right| \bar{q}_{1}\right\rangle\left[\bar{q}_{1} 1\right]\left\langle r \bar{q}_{1}\right\rangle\right)\left\langle q_{2}\left|\bar{\tau}_{\mu}\right| \bar{q}_{2}\right]
$$

$=\frac{i g_{s}^{3}}{s_{1 \bar{q}_{1}} s_{q_{2} \bar{q}_{2}}\langle r 1\rangle}\left[q_{1} \bar{q}_{2}\right]\left\langle q_{2} \bar{q}_{1}\right\rangle\left[\bar{q}_{1} 1\right]\left\langle r \bar{q}_{1}\right\rangle$, where, in the first line, we collected the prefactors and denominators from propagators, and wrote down the spinor expression for each fermion line. The spinor expressions begin with the quark, have a $\tau^{\mu}$ or $\bar{\tau}^{\mu}$ for each vertex, and a $\not p$ or $\not p$ for each fermion propagator. We expand out the propagator momentum and contract polarization vectors with a $\tau$ where possible. In the second line we rewrote the slashed propagator momenta and the slashed polarization vector in terms of spinors. Finally, we used [11] $=0$, and utilized the Fierz identity between $\tau$ and $\bar{\tau}$ to write the result in terms of spinor inner products.

Within the chirality-flow formalism, we collect scalar factors and write down the result immediately (first without arrows)
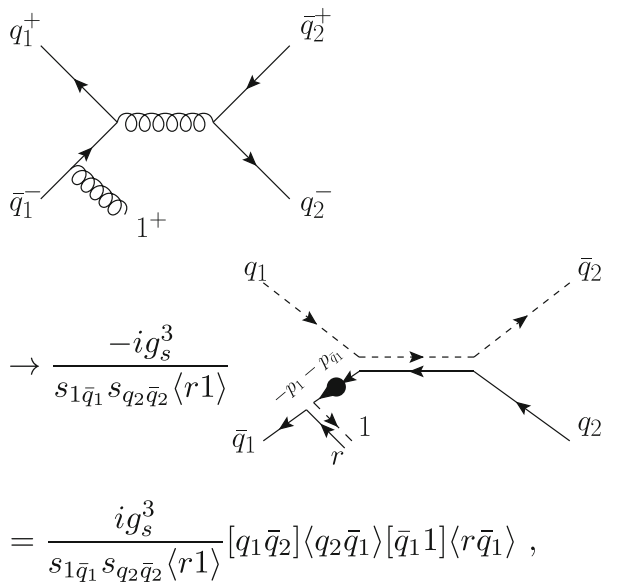

in close resemblance to the first term in (6.8).

Following the standard spinor-helicity procedure, as in (6.9), the diagram in Fig. 1c gives 


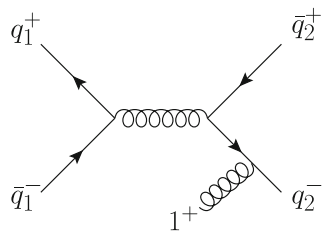

$\rightarrow \frac{-i g_{s}^{3}}{s_{1 q_{2}} s_{q_{1} \bar{q}_{1}}}\left[q_{1}\left|\tau^{\mu}\right| \bar{q}_{1}\right\rangle\left\langle q_{2}\left|\bar{\epsilon}_{1}^{+}\right|\left(\not \not_{1}+\not q_{q_{2}}\right)\left|\bar{\tau}_{\mu}\right| \bar{q}_{2}\right]$

$=\frac{-i g_{s}^{3}}{s_{1 q_{2}} s_{q_{1} \bar{q}_{1}}\langle r 1\rangle}\left[q_{1}\left|\tau^{\mu}\right| \bar{q}_{1}\right\rangle \times$

$\left(\left\langle q_{2} r\right\rangle[11]\left\langle 1\left|\bar{\tau}_{\mu}\right| \bar{q}_{2}\right]+\left\langle q_{2} r\right\rangle\left[1 q_{2}\right]\left\langle q_{2}\left|\bar{\tau}_{\mu}\right| \bar{q}_{2}\right]\right)$

$=\frac{-i g_{s}^{3}}{s_{1 q_{2}} s_{q_{1} \bar{q}_{1}}\langle r 1\rangle}\left[q_{1} \bar{q}_{2}\right]\left\langle q_{2} \bar{q}_{1}\right\rangle\left[1 q_{2}\right]\left\langle q_{2} r\right\rangle$,

which requires a few steps. However, in the chirality-flow formalism, we immediately write this down (cf. (6.7))
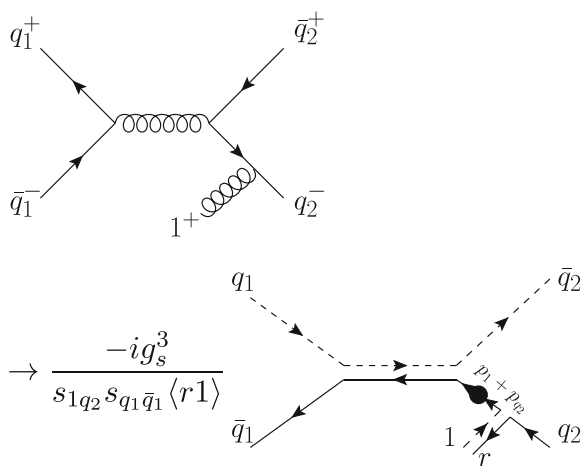

$=\frac{-i g_{s}^{3}}{s_{1 q_{2}} s_{q_{1} \bar{q}_{1}}\langle r 1\rangle}\left[q_{1} \bar{q}_{2}\right]\left\langle q_{2} r\right\rangle\left[1 q_{2}\right]\left\langle q_{2} \bar{q}_{1}\right\rangle$.

The new type of diagram in this example is Fig. 1b. Using the standard spinor-helicity method we get

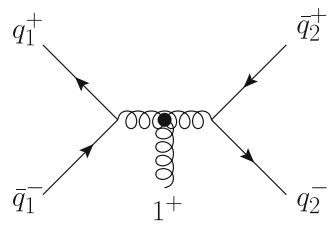

$\rightarrow \frac{i g_{s}^{3}}{\sqrt{2} s_{q_{1} \bar{q}_{1}} s_{q_{2} \bar{q}_{2}}}\left[q_{1}\left|\tau^{\mu}\right| \bar{q}_{1}\right\rangle\left\langle q_{2}\left|\bar{\tau}^{\nu}\right| \bar{q}_{2}\right]\left(\epsilon_{1}^{+}\right)^{\rho} \times$

$\times\left(g_{\mu \nu}\left(p_{q_{1}}+p_{\bar{q}_{1}}-\left(p_{q_{2}}+p_{\bar{q}_{2}}\right)\right)_{\rho}\right.$

$\left.+g_{\nu \rho}\left(p_{q_{2}}+p_{\bar{q}_{2}}-p_{1}\right)_{\mu}+g_{\rho \mu}\left(p_{1}-\left(p_{q_{1}}+p_{\bar{q}_{1}}\right)\right)_{\nu}\right)$

$=\frac{i g_{s}^{3}}{\sqrt{2} s_{q_{1} \bar{q}_{1}} s_{q_{2} \bar{q}_{2}}}\left(\left[q_{1}\left|\tau^{\mu}\right| \bar{q}_{1}\right\rangle\left\langle q_{2}\left|\bar{\tau}_{\mu}\right| \bar{q}_{2}\right] \epsilon_{1}^{+} \cdot\left(2\left(p_{q_{1}}+p_{\bar{q}_{1}}\right)+p_{1}\right)\right.$

$+\frac{1}{\sqrt{2}}\left[q_{1}\left|\left(-2 \not p_{1}-\not p_{q_{1}}-\not \bar{q}_{\bar{q}_{1}}\right)\right| \bar{q}_{1}\right\rangle\left\langle q_{2}\left|\bar{\epsilon}_{1}^{+}\right| \bar{q}_{2}\right]$

$\left.+\frac{1}{\sqrt{2}}\left[q_{1}\left|\epsilon_{1}^{+}\right| \bar{q}_{1}\right\rangle\left\langle q_{2}\left|\left(2 \bar{p}_{1}+\bar{p}_{q_{2}}+\bar{p}_{\bar{q}_{2}}\right)\right| \bar{q}_{2}\right]\right)$

$=\frac{i g_{s}^{3}}{s_{q_{1} \bar{q}_{1}} s_{q_{2} \bar{q}_{2}}\langle r 1\rangle}\left(\left[q_{1} \bar{q}_{2}\right]\left\langle q_{2} \bar{q}_{1}\right\rangle\left[1\left|\left(\not p_{q_{1}}+\not \phi_{\bar{q}_{1}}\right)\right| r\right\rangle\right.$

$\left.-\left[q_{1}\left|\not p_{1}\right| \bar{q}_{1}\right\rangle\left\langle q_{2} r\right\rangle\left[1 \bar{q}_{2}\right]+\left[q_{1} 1\right]\left\langle r \bar{q}_{1}\right\rangle\left\langle q_{2}\left|\bar{p}_{1}\right| \bar{q}_{2}\right]\right)$

$=\frac{i g_{s}^{3}}{s_{q_{1} \bar{q}_{1}} s_{q_{2} \bar{q}_{2}}\langle r 1\rangle} \times$

$\left(\left[q_{1} \bar{q}_{2}\right]\left\langle q_{2} \bar{q}_{1}\right\rangle\left[1 q_{1}\right]\left\langle q_{1} r\right\rangle+\left[q_{1} \bar{q}_{2}\right]\left\langle q_{2} \bar{q}_{1}\right\rangle\left[1 \bar{q}_{1}\right]\left\langle\bar{q}_{1} r\right\rangle\right.$

$\left.-\left[q_{1} 1\right]\left\langle 1 \bar{q}_{1}\right\rangle\left\langle q_{2} r\right\rangle\left[1 \bar{q}_{2}\right]+\left[q_{1} 1\right]\left\langle r \bar{q}_{1}\right\rangle\left\langle q_{2} 1\right\rangle\left[1 \bar{q}_{2}\right]\right)$,

where in the second step we used momentum conservation within a triple gluon vertex, $p_{a}-p_{b}=2 p_{a}+p_{c}=$ $-p_{c}-2 p_{a}$. This was done to simplify the result, since in the third step we used the Dirac equation to remove the terms containing $p_{c}$. In this step we also used the Fierz identity between $\tau$ and $\bar{\tau}$ and rewrote the polarization vector in terms of spinors. Finally, in the last step, we expanded the slashed propagator momenta in terms of spinors.

To calculate Fig. 1b using the chirality-flow method is again simpler. For the triple-gluon vertex, we start with the term in which the two quark pairs are contracted with the metric, and then add the cyclic permutations. Performing steps 1 and 2 gives 


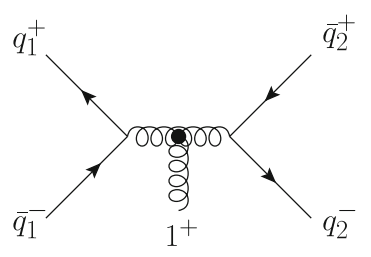

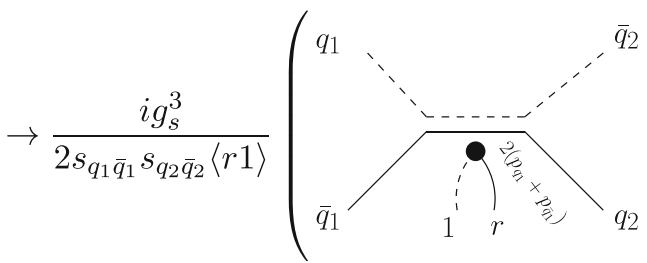

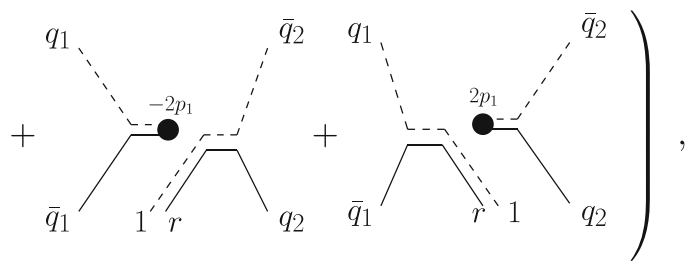

(6.14)

where, as in (6.13), we used momentum conservation and antisymmetry of the spinor inner product to simplify the slashed momenta (i.e. the labels of the momentum-dots).

Step 3 is to assign the arrows. We begin with the first chirality-flow diagram, and choose the arrow of $q_{1}$ to point inward. As before, this uniquely fixes the arrows of the dotted line to point from $q_{1} \rightarrow \bar{q}_{2}$, and the solid line from $q_{2} \rightarrow \bar{q}_{1}$. However, the spinor line from $1 \leftrightarrow r$ is unaffected by this arrow choice. In this sense, we say that the chirality-flow diagram has two disconnected pieces, and we are free to choose the arrow to e.g. point from $1 \rightarrow r$. Similarly, in the second and third terms, the arrow directions of $q_{1}$ and $\bar{q}_{2}$ can be chosen independently of each other. An appropriate set of arrow choices gives the spinor structure
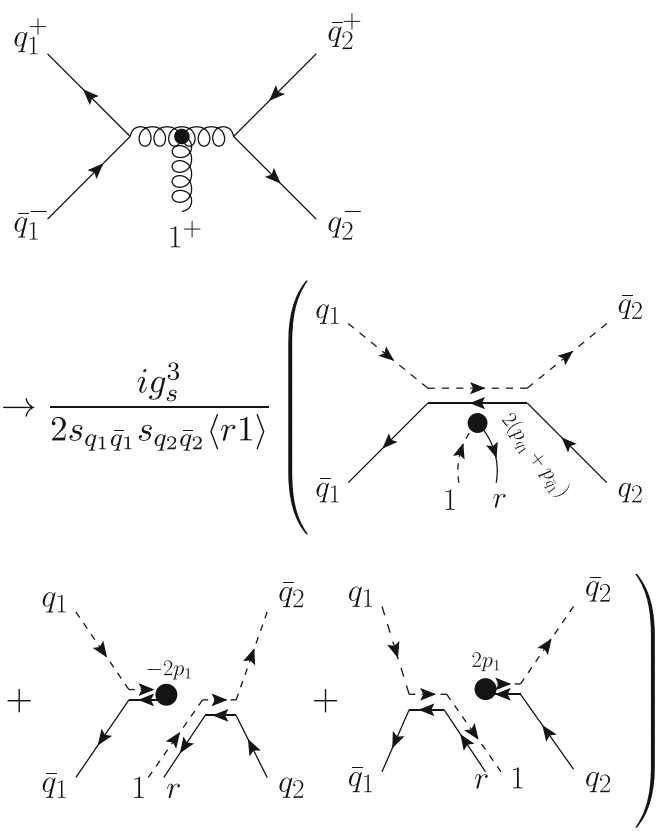

$=\frac{i g_{s}^{3}}{s_{q_{1} \bar{q}_{1}} s_{q_{2} \bar{q}_{2}}\langle r 1\rangle}\left(\left[q_{1} \bar{q}_{2}\right]\left\langle q_{2} \bar{q}_{1}\right\rangle\left(\left[1 q_{1}\right]\left\langle q_{1} r\right\rangle+\left[1 \bar{q}_{1}\right]\left\langle\bar{q}_{1} r\right\rangle\right)\right.$
$\left.-\left[q_{1} 1\right]\left\langle 1 \bar{q}_{1}\right\rangle\left\langle q_{2} r\right\rangle\left[1 \bar{q}_{2}\right]+\left[q_{1} 1\right]\left\langle r \bar{q}_{1}\right\rangle\left\langle q_{2} 1\right\rangle\left[1 \bar{q}_{2}\right]\right)$

as in (6.13).

Comparing the two methods, we see that the chirality-flow method simplifies the calculation in two ways. First, there is no need to explicitly write all objects as spinor expressions, and then use the Fierz identity to remove the remaining Lorentz indices. Second, it is much more transparent which spinor inner products occur.

Finally, we stress that once one is familiar with the flow formalism, it is possible to write down an amplitude like this in one step, 


$$
M\left(0 \rightarrow q_{2}^{-} 1^{+} \bar{q}_{1}^{-} q_{1}^{+} \bar{q}_{2}^{+}\right)
$$$$
=-\frac{i g_{s}^{3}}{\langle r 1\rangle}\left(\frac{1}{s_{1 \bar{q}_{1}} s_{q_{2} \bar{q}_{2}}}\right.
$$
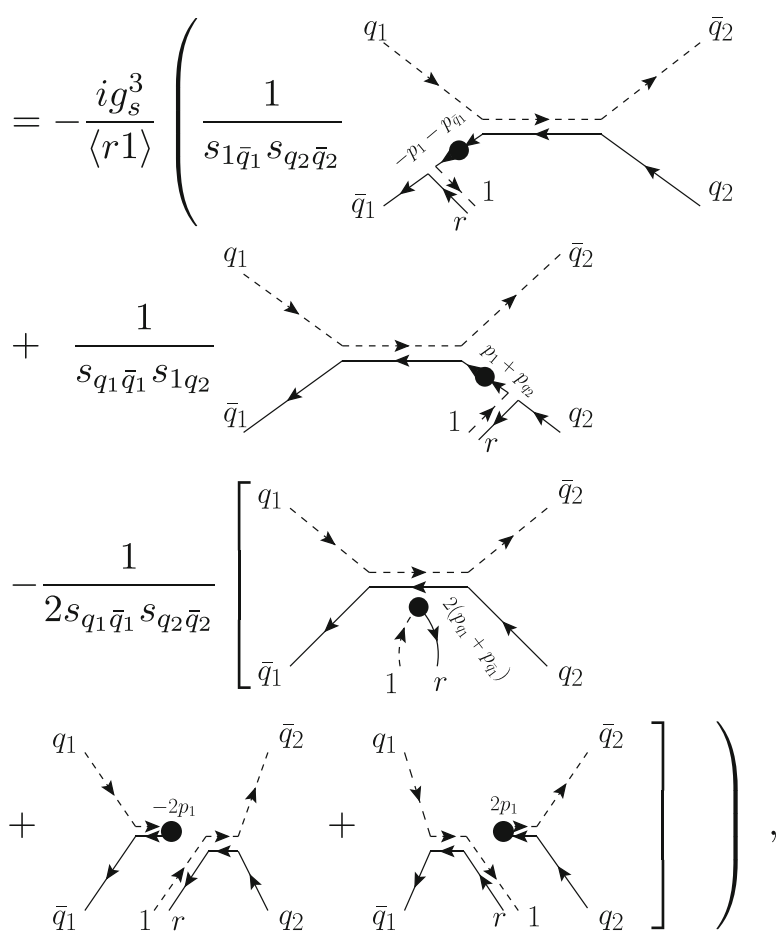

where the first two terms correspond to the diagrams in Fig. 1a and $1 \mathrm{c}$ respectively, and the last three terms to the diagram in Fig. 1b. We again emphasize that the above already contains the sum of all spinor inner products, and that we may simplify the result by choosing the reference momentum appropriately to generate spinor inner products of the form $\langle i i\rangle$ or $[i i]$.

\section{$6.4 q \bar{q} \rightarrow g g$}

For this example we will leave the helicity of the vector bosons unassigned. We call this the helicity-agnostic case. This can be done in the traditional spinor-helicity method as well, but its consequences are more obvious in the chiralityflow picture, as the helicity and chirality structures of the diagrams are more transparent. To this end, we introduce a common notation for the bispinor representations of the negativeand positive-helicity polarization vectors in Eqs. $(3.32,3.33)$ and Eqs. (3.36, 3.37),

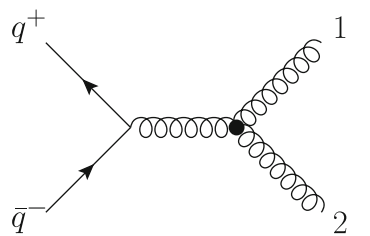

(a)

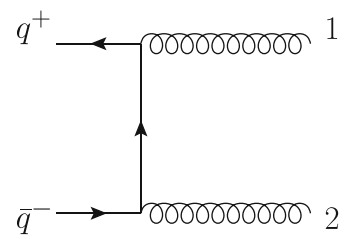

(b)
Fig. 2 The two diagrams contributing to the partial helicity amplitude $M\left(0 \rightarrow q^{+} 12 \bar{q}^{-}\right)$, multiplying the color factor $t_{q i}^{1} t_{i \bar{q}}^{2}$

$$
\begin{gathered}
\epsilon_{h}^{\dot{\beta} \alpha}\left(p_{i}, r\right)=\frac{\left.\mid i_{p}\right]\left\langle i_{m}\right|}{f_{h}\left(i_{p}, i_{m}\right)}=\frac{1}{f_{h}\left(i_{p}, i_{m}\right)} \bigcirc{ }^{i_{m}}, \\
\bar{\epsilon}_{h, \beta \dot{\alpha}}\left(p_{i}, r\right)=\frac{\left|i_{m}\right\rangle\left[i_{p} \mid\right.}{f_{h}\left(i_{p}, i_{m}\right)}=\frac{1}{f_{h}\left(i_{p}, i_{m}\right)} \\
\stackrel{-\cdots-i_{p}}{i_{m}},
\end{gathered}
$$

where $h=\mp$,

$$
\begin{gathered}
i_{p} \text { and } i_{m}=\left\{\begin{array}{ll}
r \text { and } i, & h=- \\
i \text { and } r, & h=+
\end{array},\right. \\
f_{h}\left(i_{p}, i_{m}\right)=\left\{\begin{array}{ll}
{\left[i_{m} i_{p}\right]=[i r],} & h=- \\
\left\langle i_{m} i_{p}\right\rangle=\langle r i\rangle, & h=+
\end{array},\right.
\end{gathered}
$$

and where $i_{p}\left(i_{m}\right)$ denotes the positive- (negative-)helicity spinor in gluon $i$.

For this example we consider the partial helicity amplitude $M\left(0 \rightarrow q^{+} 12 \bar{q}^{-}\right)$, multiplying the color factor $t_{q i}^{1} t_{i \bar{q}}^{2}$. This means that we only consider the two diagrams in Fig. 2. For the Lorentz structure of the diagram in Fig. $2 b$ we get

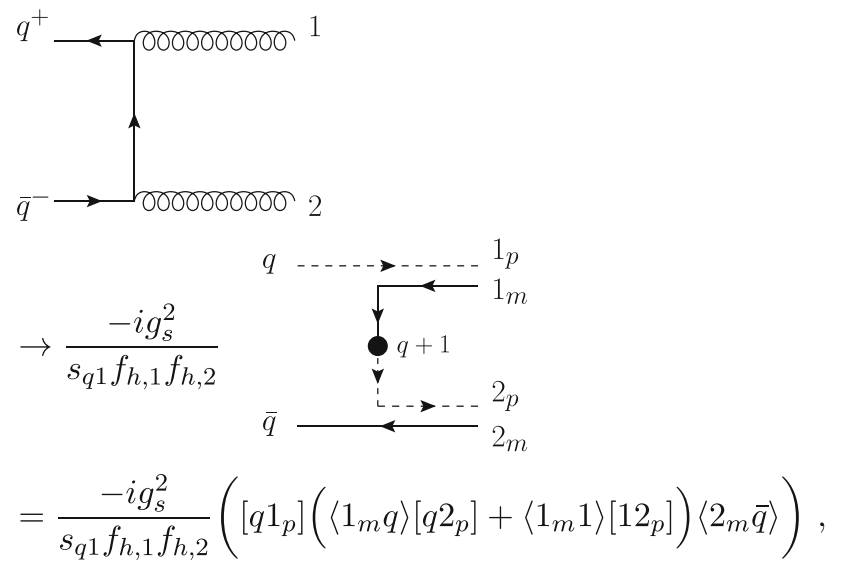


while for the diagram in Fig. 2a we obtain
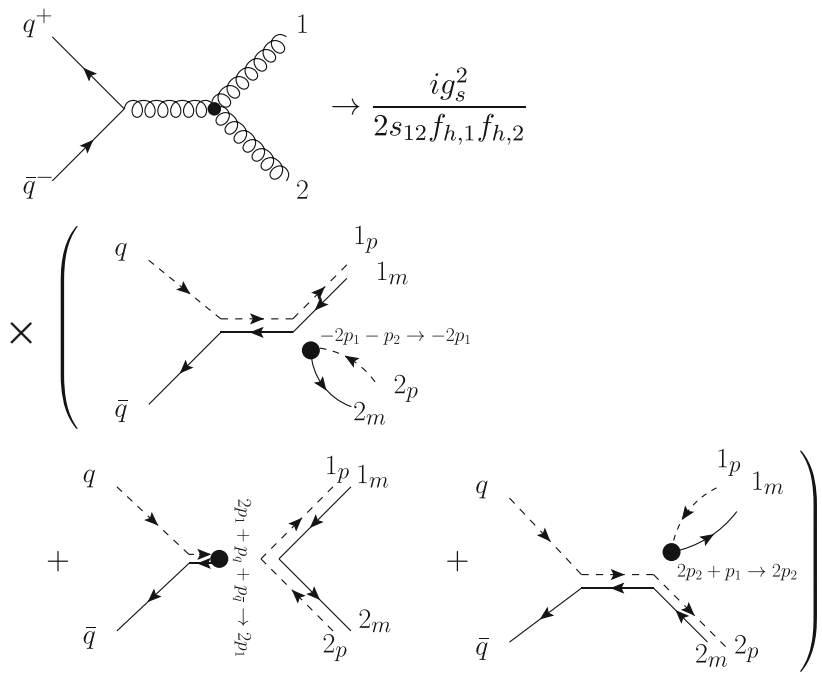

$=\frac{i g_{s}^{2}}{s_{12} f_{h, 1} f_{h, 2}}\left(-\left[q 1_{p}\right]\left\langle 1_{m} \bar{q}\right\rangle\left[2_{p} 1\right]\left\langle 12_{m}\right\rangle\right.$

$\left.+\left\langle 1_{m} 2_{m}\right\rangle\left[2_{p} 1_{p}\right][q 1]\langle 1 \bar{q}\rangle+\left[q 2_{p}\right]\left\langle 2_{m} \bar{q}\right\rangle\left[1_{p} 2\right]\left\langle 21_{m}\right\rangle\right)$,

where we again used momentum conservation to rewrite all momenta in the triple-gluon vertex $p_{a}-p_{b}=2 p_{a}+p_{c}=$ $-2 p_{b}-p_{c}$. Since $p_{c}=p_{2}$ in the first flow diagram, and $p_{c}=p_{1}$ in the last diagram, and in each of these either $i_{m}=i$ or $i_{p}=i$ for $i=1,2$, we could again remove the term with $p_{c}$. The full result for a gluon of either helicity is then

$$
\begin{aligned}
M(0 & \left.\rightarrow q^{+} 12 \bar{q}^{-}\right)=\frac{i g_{s}^{2}}{f_{h, 1} f_{h, 2}}\left(\frac { 1 } { s _ { 1 2 } } \left[-\left[q 1_{p}\right]\left\langle 1_{m} \bar{q}\right\rangle\left[2_{p} 1\right]\left\langle 12_{m}\right\rangle\right.\right. \\
& \left.+\left\langle 1_{m} 2_{m}\right\rangle\left[2_{p} 1_{p}\right][q 1]\langle 1 \bar{q}\rangle+\left[q 2_{p}\right]\left\langle 2_{m} \bar{q}\right\rangle\left[1_{p} 2\right]\left\langle 21_{m}\right\rangle\right] \\
& \left.-\frac{1}{s_{q 1}}\left[q 1_{p}\right]\left(\left\langle 1_{m} q\right\rangle\left[q 2_{p}\right]+\left\langle 1_{m} 1\right\rangle\left[12_{p}\right]\right)\left\langle 2_{m} \bar{q}\right\rangle\right)
\end{aligned}
$$

If both gluons have positive helicity, then $\left(1_{m}, 1_{p}\right)=$ $\left(r_{1}, 1\right)$ and $\left(2_{m}, 2_{p}\right)=\left(r_{2}, 2\right)$ such that choosing $1_{m}=$ $2_{m}=\bar{q}$ and using the antisymmetry of the spinor inner products we see that the amplitude vanishes. If both gluons have negative helicity, choosing $1_{p}=2_{p}=q$ we see that the amplitude vanishes. Looking at the chirality-flow diagrams in Eqs. (6.19) and (6.20), this is easy to see from an early stage, since e.g. $q$ always flows to/from either $1_{p}$ or $2_{p}$, except for when it flows to/from $\bar{q}$, in which case $2_{p}$ flows to/from $1_{p}$.

The only non-zero case is the MHV case, where e.g. $h_{1}=$ ,$- h_{2}=+,\left(1_{m}, 1_{p}\right)=\left(1, r_{1}\right)$ and $\left(2_{m}, 2_{p}\right)=\left(r_{2}, 2\right)$. We choose $1_{p}=q$ and $2_{m}=\bar{q}$, such that the reference momentum of each gluon is equal to the momentum of the quark with opposite helicity, giving

$$
\begin{aligned}
M & \left(0 \rightarrow q^{+} 1^{-} 2^{+} \bar{q}^{-}\right) \\
& =\frac{i g_{s}^{2}}{[1 q]\langle\bar{q} 2\rangle}\left[\frac{1}{s_{12}}[-0+\langle 1 \bar{q}\rangle[2 q][q 1]\langle 1 \bar{q}\rangle+0]-\frac{1}{s_{q 1}}(0)\right] \\
& =-\frac{i g_{s}^{2}\langle\bar{q} 1\rangle^{2}[2 q]}{\langle\bar{q} 2\rangle\langle 12\rangle[21]} \frac{\langle\bar{q} q\rangle\langle q 1\rangle}{\langle\bar{q} q\rangle\langle q 1\rangle}=-\frac{i g_{s}^{2}\langle\bar{q} 1\rangle^{2}\langle q 1\rangle(-[21]\langle 1 \bar{q}\rangle)}{\langle q 1\rangle\langle 12\rangle\langle 2 \bar{q}\rangle\langle\bar{q} q\rangle[21]} \\
& =-\frac{i g_{s}^{2}\langle\bar{q} 1\rangle^{3}\langle q 1\rangle}{\langle q 1\rangle\langle 12\rangle\langle 2 \bar{q}\rangle\langle\bar{q} q\rangle},
\end{aligned}
$$

which is the standard MHV formula. ${ }^{19}$ To obtain this, in the second line, we multiplied by one, expanded out $s_{12}=$ $\langle 12\rangle[21]$ (see (A.37)) and canceled the [q1] brackets, before using that $[2 q]\langle q \bar{q}\rangle=-[21]\langle 1 \bar{q}\rangle$, due to momentum conservation (see (A.39)).

$6.5 g g \rightarrow g g$

In this example, we explore the four-gluon vertex. We consider the three diagrams contributing to the partial helicity amplitude $M\left(0 \rightarrow 1^{h_{1}} 2^{h_{2}} 3^{h_{3}} 4^{h_{4}}\right)$, multiplying the color factor $\operatorname{Tr}\left(t^{1} t^{2} t^{3} t^{4}\right)$. For now, we leave the helicities of each particle unassigned. Steps 1 and 2 give for the s-channel diagram
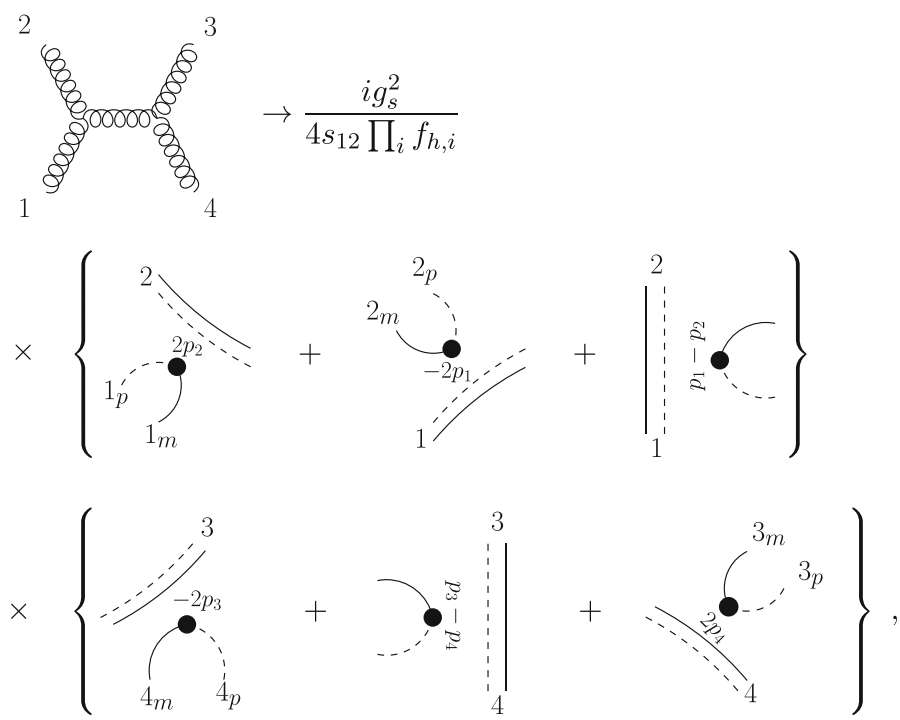

$(6.23)$

where we have factorized the two triple-gluon vertices, used the shorthand notation $i=\left(i_{m}, i_{p}\right)$, and written the gluon propagator as a double line without a label. Stitch-

19 The minus sign here is often omitted in the literature [60], such that this MHV QCD amplitude resembles the relevant gluino-gluon amplitude, and therefore obeys the supersymmetric Ward identities without additional minus signs $[52,53,55]$. 


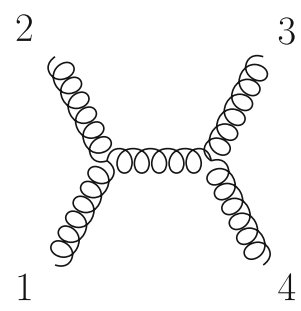

(a)

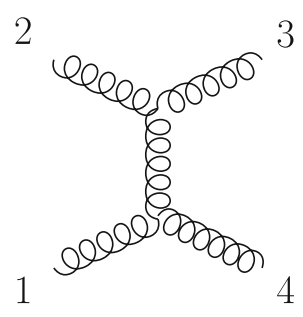

(b)

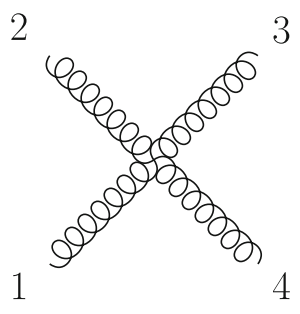

(c)

Fig. 3 The three diagrams contributing to the color-partial helicity amplitude $M\left(0 \rightarrow 1^{h_{1}} 2^{h_{2}} 3^{h_{3}} 4^{h_{4}}\right)$

ing together the two factorized terms and assigning arrows results in

The t-channel diagram Fig. 3b has the same form as the s-channel diagram, and can be found by permuting the labels

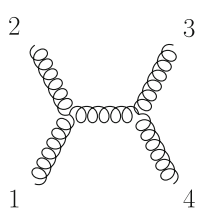

$\rightarrow \frac{i g_{s}^{2}}{4 s_{12} \prod_{i} f_{h, i}}$
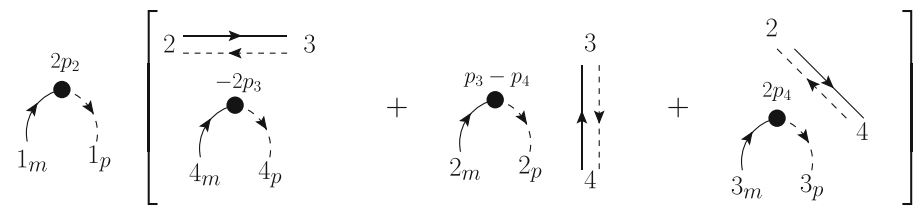

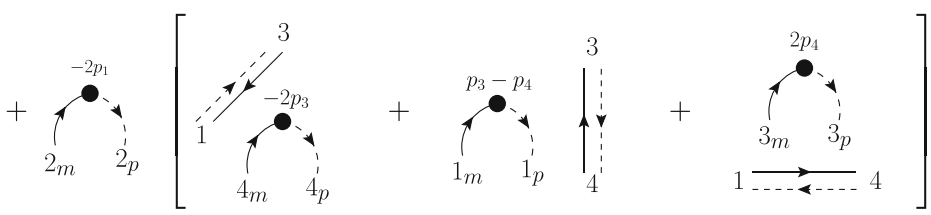

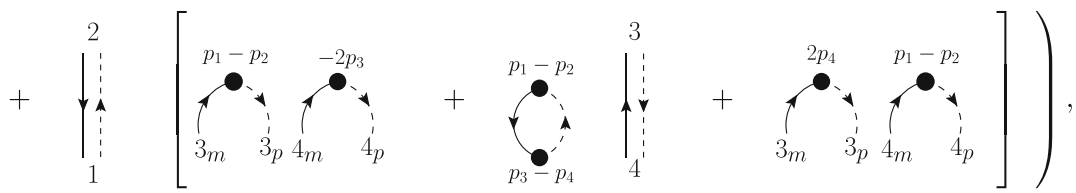

where we see a contraction of momenta

$\bigodot_{p_{j}}^{p_{i}} \bigwedge_{i}^{\prime}=2 p_{i} \cdot p_{j} \stackrel{p_{i}^{2}=p_{j}^{2}=0}{=}\langle i j\rangle[j i]$

for the first time.
$(1,2,3,4)$ in the cyclic direction, $1 \rightarrow 2,2 \rightarrow 3$, etc. What remains to calculate is therefore only the contact diagram, Fig. 3c, for which we directly write

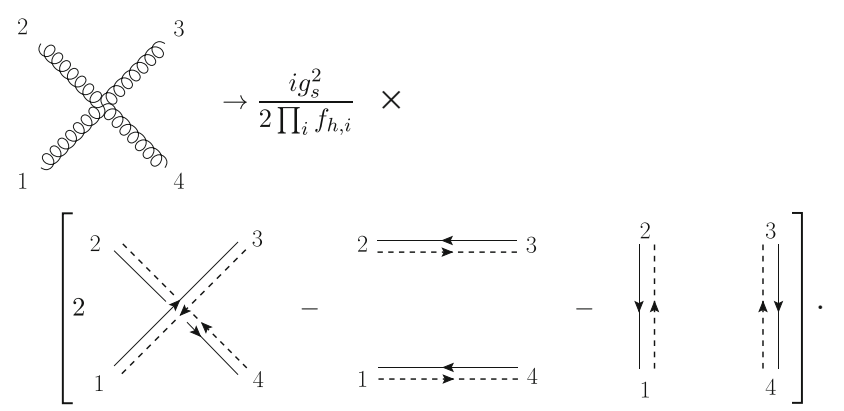

(6.26) 
While the sum of the diagrams still appears complicated, it contains all of the information needed to obtain the fourgluon scattering amplitude for any combination of gluon helicities. For example, if we choose $h_{1}=h_{2}=-h_{3}=$ $-h_{4}=1, r_{1}=r_{2}=p_{4}$ and $r_{3}=r_{4}=p_{1}$, only a single term, the first term in (6.24), remains. The resulting amplitude is then

$$
\begin{aligned}
& M\left(1^{+}, 2^{+}, 3^{-}, 4^{-}\right)=-i g_{s}^{2} \frac{\langle 34\rangle^{2}[21]^{2}}{s_{12}\langle 14\rangle[41]} \\
& =-i g_{s}^{2} \frac{\langle 34\rangle^{2}[21]}{\langle 12\rangle\langle 14\rangle[41]} \frac{\langle 34\rangle^{2}}{\langle 34\rangle^{2}}=i g_{s}^{2} \frac{\langle 34\rangle^{4}}{\langle 12\rangle\langle 34\rangle\langle 41\rangle} \frac{[21]}{\langle 34\rangle[41]} \\
& =i g_{s}^{2} \frac{\langle 34\rangle^{4}}{\langle 12\rangle\langle 34\rangle\langle 41\rangle} \frac{[21]}{-\langle 32\rangle[21]}=i g_{s}^{2} \frac{\langle 34\rangle^{4}}{\langle 12\rangle\langle 23\rangle\langle 34\rangle\langle 41\rangle},
\end{aligned}
$$

as expected $[8,54]$.

\section{Conclusion and outlook}

In this paper we have presented a new graphical formalism for calculating massless QED and QCD Feynman diagrams.

In the spinor-helicity formalism, Dirac matrices are abandoned in favor of the smaller Pauli matrices, and in the Weyl-van-der-Waerden formalism further simplification is obtained by recasting Feynman rules and diagrams to expressions without Pauli matrices, which instead depend on the antisymmetric $\epsilon$-tensor, i.e., the $\operatorname{SL}(2, C)$ invariant object.

In this work, we take this one step further, and argue that we can directly write diagrams, and hence amplitudes, as a combination of spinor inner products. Using charge conjugation, we argue that we can formulate a set of chiralityflow Feynman rules for massless QED and QCD diagrams at tree-level. After this, there are no algebraic manipulations or matrix equations involved in the process of finding the spinor inner products appearing in a scattering amplitude.

Conceptually our method is similar to the idea of a flow of color in QCD, in the sense that after stripping off the external color/spinor wave functions, what remains can be thought of as Kronecker contractions connecting the external color/spinor structures. We therefore dub our method the chirality-flow formalism. We remark, however, that the flow picture for color and the flow picture for chirality can be used completely independently, and in fact we have given both ordinary Feynman rules and color-flow Feynman rules for the chirality-flow method.

We also note that the methods differ by using one su(3)algebra for color and two (complexified) su(2)-algebras for chirality, hence we need two different types of lines - dotted and undotted - which can never be contracted with each other, since the corresponding object would not be Lorentz invariant.

On the more practical side we note that, aside from being more transparent, the chirality-flow method also shortens actual calculations by removing a few steps compared to the ordinary spinor-helicity method. This complete avoidance of matrix structure may turn out beneficial in automated tools for calculating amplitudes, particularly those relying on Feynman diagrams, e.g. [74-76].

Finally, we remark that while the present paper deals only with massless particles in QED and QCD, the spinor-helicity formalism for massive particles is well known, and work towards completing the chirality-flow method for the full standard model is ongoing.

Acknowledgements We thank Johan Bijnens and Rikkert Frederix for constructive feedback on the manuscript. This work was supported by the Swedish Research Council (contract numbers 2012-02744 and 2016-05996), as well as the European Union's Horizon 2020 research and innovation programme (Grant agreement no 668679). This work has also received funding from the European Union's Horizon 2020 research and innovation programme as part of the Marie SklodowskaCurie Innovative Training Network MCnetITN3 (Grant agreement no. 722104).

Data Availability Statement This manuscript has no associated data or the data will not be deposited. [Authors' comment: This manuscript concerns theoretical work and thus has no associated data.]

Open Access This article is licensed under a Creative Commons Attribution 4.0 International License, which permits use, sharing, adaptation, distribution and reproduction in any medium or format, as long as you give appropriate credit to the original author(s) and the source, provide a link to the Creative Commons licence, and indicate if changes were made. The images or other third party material in this article are included in the article's Creative Commons licence, unless indicated otherwise in a credit line to the material. If material is not included in the article's Creative Commons licence and your intended use is not permitted by statutory regulation or exceeds the permitted use, you will need to obtain permission directly from the copyright holder. To view a copy of this licence, visit http://creativecomm ons.org/licenses/by/4.0/.

Funded by $\mathrm{SCOAP}^{3}$.

\section{A Conventions and identities}

In this appendix, we give some conventions and collect some additional identities, as well as explicit representations for spinors and four-vectors in the spinor representation. For convenience, some of the previous definitions and identities will be repeated in this appendix.

\section{A.1 Pauli matrices}

We define the Dirac matrices in the chiral, or Weyl, representation as

$\gamma^{\mu}=\left(\begin{array}{cc}0 & \sigma^{\mu} \\ \bar{\sigma}^{\mu} & 0\end{array}\right)=\left(\begin{array}{cc}0 & \sqrt{2} \tau^{\mu} \\ \sqrt{2} \bar{\tau}^{\mu} & 0\end{array}\right)$, 
and the Pauli matrices as

$$
\begin{aligned}
\sigma^{\mu} & =\left(\sigma^{0}, \sigma\right)=\left(\sigma^{0}, \sigma^{1}, \sigma^{2}, \sigma^{3}\right) \\
& =\left(\left(\begin{array}{ll}
1 & 0 \\
0 & 1
\end{array}\right),\left(\begin{array}{ll}
0 & 1 \\
1 & 0
\end{array}\right),\left(\begin{array}{cc}
0 & -i \\
i & 0
\end{array}\right),\left(\begin{array}{cc}
1 & 0 \\
0 & -1
\end{array}\right)\right), \\
\bar{\sigma}^{\mu} & =\left(\sigma^{0},-\sigma\right),
\end{aligned}
$$

or equivalently the Infeld-van-der-Waerden matrices, or $\tau$ matrices, as $\tau^{\mu}=\frac{1}{\sqrt{2}} \sigma^{\mu}$ and $\bar{\tau}^{\mu}=\frac{1}{\sqrt{2}} \bar{\sigma}^{\mu}$. The elements of the Pauli or $\tau$ matrices are denoted by $\tau^{\mu, \dot{\alpha} \beta}=\frac{1}{\sqrt{2}} \sigma^{\mu, \dot{\alpha} \beta}$ and $\bar{\tau}_{\alpha \dot{\beta}}^{\mu}=\frac{1}{\sqrt{2}} \bar{\sigma}_{\alpha \dot{\beta}}^{\mu}$. The Pauli matrices are Hermitian $2 \times 2$ matrices, i.e. $\left(\sigma^{\mu}\right)^{\dagger}=\sigma^{\mu}$, and we further have $\left(\sigma^{\mu}\right)^{2}=$ $\left(\begin{array}{ll}1 & 0 \\ 0 & 1\end{array}\right)$, for $\mu=0,1,2,3$. Consequently, $\left(\tau^{\mu}\right)^{\dagger}=\tau^{\mu}$ and $\left(\tau^{\mu}\right)^{2}=\frac{1}{2}\left(\begin{array}{ll}1 & 0 \\ 0 & 1\end{array}\right)$, for $\mu=0,1,2,3$. The normalization of the $\tau$ matrices is chosen such that no unnecessary powers of 2 are carried around in the algebraic relations,

$$
\begin{aligned}
\operatorname{Tr}\left(\tau^{\mu} \bar{\tau}^{v}\right) & =g^{\mu \nu}, \\
\operatorname{Tr}\left(\bar{\tau}^{\mu} \bar{\tau}^{v}\right) & =\delta^{\mu \nu}, \\
\operatorname{Tr}\left(\tau^{\mu} \tau^{\nu}\right) & =\delta^{\mu \nu}, \\
\tau_{\mu}^{\dot{\alpha} \beta} \bar{\tau}_{\gamma \dot{\eta}}^{\mu} & =\delta_{\gamma}^{\beta} \delta_{\dot{\eta}}^{\dot{\alpha}}, \\
\bar{\tau}_{\alpha \dot{\beta}}^{\mu} \bar{\tau}_{\mu, \gamma \dot{\eta}} & =\epsilon_{\alpha \gamma} \epsilon_{\dot{\beta} \dot{\eta}}, \\
\tau^{\mu, \dot{\alpha} \beta} \tau_{\mu}^{\dot{\gamma} \eta} & =\epsilon^{\dot{\alpha} \dot{\gamma}} \epsilon^{\beta \eta},
\end{aligned}
$$

where $g^{\mu \nu}=\operatorname{diag}(1,-1,-1,-1)$ denotes the Minkowski metric and $\epsilon$ the Levi-Civita tensor (see (3.10)).

\section{A.2 Spinors and spinor inner products}

We recall from Sect. 3.1 that the Weyl spinors, i.e. the twocomponent spinors in the chiral or Weyl representation, are solutions to the Weyl equations, i.e. the decoupled equations for left- and right-chiral two-component spinors that follow from the Dirac equation in the massless case. We denote the Weyl spinors of massless outgoing left-chiral (positivehelicity) fermions of momentum $p$ by $\tilde{\lambda}_{\dot{\alpha}}(p)=\tilde{\lambda}_{p, \dot{\alpha}}$ and outgoing left-chiral (positive-helicity) antifermions $\tilde{\lambda}^{\dot{\alpha}}(p)=$ $\tilde{\lambda}{ }_{p}^{\dot{\alpha}}$, while right-chiral (negative-helicity) Weyl spinors of massless outgoing fermions and antifermions of momentum $p$ are denoted by $\lambda^{\alpha}(p)=\lambda_{p}^{\alpha}$ and $\lambda_{\alpha}(p)=\lambda_{p, \alpha}$ respectively.

Explicit representations of the (massless) Weyl spinors are for example,

$$
\begin{aligned}
\lambda_{p}^{\alpha} \leftrightarrow\langle p| & =\frac{1}{\sqrt{p^{+}}}\left(p^{\perp},-p^{+}\right), \\
\lambda_{p, \alpha} \leftrightarrow|p\rangle & =\frac{1}{\sqrt{p^{+}}}\left(\begin{array}{l}
p^{+} \\
p^{\perp}
\end{array}\right),
\end{aligned}
$$

$$
\begin{aligned}
\tilde{\lambda}_{p, \dot{\alpha}} \leftrightarrow[p \mid & =\frac{1}{\sqrt{p^{+}}}\left(p^{+}, p^{\perp^{*}}\right), \\
\left.\tilde{\lambda}_{p}^{\dot{\alpha}} \leftrightarrow \mid p\right] & =\frac{1}{\sqrt{p^{+}}}\left(\begin{array}{c}
p^{\perp^{*}} \\
-p^{+}
\end{array}\right),
\end{aligned}
$$

where we used light-cone coordinates ${ }^{20}$,

$p^{ \pm}=p^{0} \pm p^{3}, \quad p^{\perp}=p^{1}+i p^{2}, \quad p^{\perp^{*}}=p^{1}-i p^{2}$.

The Weyl spinors are related to each other by Hermitian conjugation,

$|p\rangle^{\dagger}=[p \mid \text { and } \mid p]^{\dagger}=\langle p|$,

or in case of the components $\left(\lambda_{p, \alpha}\right)^{*}=\left(\tilde{\lambda}_{p, \dot{\alpha}}\right)$ and $\left(\tilde{\lambda}_{p}^{\dot{\alpha}}\right)^{*}=$ $\left(\lambda_{p}^{\alpha}\right)$ for $\alpha=\dot{\alpha}=1,2$, which is easily confirmed for the explicit representations in Eqs. (A.10) and (A.11).

We recall from (3.11) that spinor indices of two-component Weyl spinors are raised and lowered by the Levi-Civita tensor, ${ }^{21}$

$\lambda_{p, \alpha}=\epsilon_{\alpha \beta} \lambda_{p}^{\beta}, \quad \tilde{\lambda}_{p, \dot{\alpha}}=\epsilon_{\dot{\alpha} \dot{\beta}} \tilde{\lambda}_{p}^{\dot{\beta}}$,

$$
\lambda_{p}^{\alpha}=\epsilon^{\alpha \beta} \lambda_{p, \beta}, \quad \tilde{\lambda}_{p}^{\dot{\alpha}}=\epsilon^{\dot{\alpha} \dot{\beta}} \tilde{\lambda}_{p, \dot{\beta}}
$$

as is easily seen for the representations in Eqs. (A.10) and (A.11) using (3.10).

We also recall the antisymmetric Lorentz invariant spinor inner products, Eqs. (3.12) and (3.13),

$$
\begin{aligned}
\langle i j\rangle & =\lambda_{i}^{\alpha} \lambda_{j, \alpha}=\epsilon^{\alpha \beta} \lambda_{i, \beta} \lambda_{j, \alpha}=-\epsilon^{\beta \alpha} \lambda_{i, \beta} \lambda_{j, \alpha} \\
& =-\lambda_{i, \beta} \lambda_{j}^{\beta}=-\langle j i\rangle, \\
{[i j] } & =\tilde{\lambda}_{i, \dot{\alpha}} \tilde{\lambda}_{j}^{\dot{\alpha}}=\epsilon_{\dot{\alpha} \dot{\beta}} \tilde{\lambda}_{i}^{\dot{\beta}} \tilde{\lambda}_{j}^{\dot{\alpha}}=-\epsilon_{\dot{\beta} \dot{\alpha}} \tilde{\lambda}_{i}^{\dot{\beta}} \tilde{\lambda}_{j}^{\dot{\alpha}} \\
& =-\tilde{\lambda}_{i}^{\dot{\beta}} \tilde{\lambda}_{j, \dot{\beta}}=-[j i] .
\end{aligned}
$$

Using the explicit representations of the Weyl spinors in Eqs. (A.10) and (A.11), we have

$$
\begin{aligned}
& \langle i j\rangle=\lambda_{i}^{\alpha} \lambda_{j, \alpha}=\frac{1}{\sqrt{p_{i}^{+} p_{j}^{+}}}\left(p_{i}^{\perp} p_{j}^{+}-p_{j}^{\perp} p_{i}^{+}\right), \\
& {[i j]=\tilde{\lambda}_{i \dot{\alpha}} \tilde{\lambda}_{j}^{\dot{\alpha}}=\frac{1}{\sqrt{p_{i}^{+} p_{j}^{+}}}\left(p_{i}^{+} p_{j}^{\perp^{*}}-p_{j}^{+} p_{i}^{\perp^{*}}\right),}
\end{aligned}
$$

consistent with $[i i]=\langle j j\rangle=0$ and $\langle i j\rangle^{*}=-[i j]=[j i]$.

20 We have $p^{+} p^{-}=\left(p^{0}+p^{3}\right)\left(p^{0}-p^{3}\right)=\left(p^{0}\right)^{2}-\left(p^{3}\right)^{2}$ and $p^{\perp} p^{\perp^{*}}=\left(p^{1}+i p^{2}\right)\left(p^{1}-i p^{2}\right)=\left(p^{1}\right)^{2}+\left(p^{2}\right)^{2}$. Furthermore, $p^{2}=$ $p^{+} p^{-}-p^{\perp} p^{\perp^{*}}$, i.e. for $p^{2}=0$ we have $p^{+} p^{-}=p^{\perp} p^{\perp^{*}}$.

21 We recall from (3.10) that the Levi-Civita or $\epsilon$ tensor is defined as $\epsilon^{12}=-\epsilon^{21}=\epsilon_{21}=-\epsilon_{12}=1$, or in matrix notation as $\epsilon^{\alpha \beta}=\epsilon^{\dot{\alpha} \dot{\beta}} \leftrightarrow$ $\left(\begin{array}{cc}0 & 1 \\ -1 & 0\end{array}\right)=i \sigma^{2}$ and $\epsilon_{\alpha \beta}=\epsilon_{\dot{\alpha} \dot{\beta}} \leftrightarrow\left(\begin{array}{cc}0 & -1 \\ 1 & 0\end{array}\right)=-i \sigma^{2}$. With our definition of the $\epsilon$-tensor we have that $\epsilon_{\alpha \beta} \epsilon^{\beta \gamma}=\delta_{\alpha}^{\gamma}$ and $\epsilon^{\dot{\alpha} \dot{\beta}} \epsilon_{\dot{\beta} \dot{\gamma}}=\delta_{\dot{\gamma}}^{\dot{\alpha}}$, and that e.g. $\epsilon_{\alpha \beta} \lambda_{p}^{\beta}=-\epsilon_{\beta \alpha} \lambda_{p}^{\beta}=-\lambda_{p}^{\beta} \epsilon_{\beta \alpha}$, etc. 
We also state the Schouten identities

$$
\begin{aligned}
& \langle i j\rangle\langle k l\rangle+\langle i k\rangle\langle l j\rangle+\langle i l\rangle\langle j k\rangle \\
& \quad=\langle i|(|j\rangle\langle k l\rangle+|k\rangle\langle l j\rangle+|l\rangle\langle j k\rangle)=0, \\
& {[i j][k l]+[i k][l j]+[i l][j k]} \\
& \quad=[i|(\mid j][k l]+| k][l j]+\mid l][j k])=0,
\end{aligned}
$$

which are a consequence of the fact that any three twocomponent spinors are linearly dependent.

For the spinor transformations, we use the common notation $\left(j_{L}, j_{R}\right)$ for representations of the Lorentz algebra, corresponding to the representations of the mutually commuting generator combinations

$\mathbf{N}^{L}=\frac{1}{2}(\mathbf{J}-i \mathbf{K})$,

$\mathbf{N}^{R}=\frac{1}{2}(\mathbf{J}+i \mathbf{K})$,

where $\mathbf{J}$ and $\mathbf{K}$ denote the generators of rotations and boosts respectively and where

$\left[\mathbf{N}^{L, i}, \mathbf{N}^{R, j}\right]=0$

For left-chiral spinors $\tilde{\lambda}_{p}^{\dot{\alpha}}$ in the $\left(j_{L}, j_{R}\right)=(1 / 2,0)$ representation, we have $\mathbf{J}=-i \mathbf{K}=\sigma / 2$, i.e. $\mathbf{N}^{L}=\sigma / 2$ and $\mathbf{N}^{R}=0$. Under Lorentz transformations they transform as

$\tilde{\lambda}_{p}^{\dot{\alpha}} \rightarrow\left(\Lambda_{L}\right)_{\dot{\beta}}^{\dot{\alpha}} \tilde{\lambda}_{p}^{\dot{\beta}} \quad$ with $\quad \Lambda_{L}=e^{(-i \boldsymbol{\theta}+\boldsymbol{\eta}) \cdot \boldsymbol{\sigma} / 2}$

where $\boldsymbol{\theta}$ and $\boldsymbol{\eta}$ denote rotation angles and boost parameters. For right-chiral spinors $\lambda_{p, \beta}$ in the $\left(j_{L}, j_{R}\right)=(0,1 / 2)$ representation, we similarly have $\mathbf{J}=i \mathbf{K}=\sigma / 2$, i.e. $\mathbf{N}^{L}=0$ and $\mathbf{N}^{R}=\sigma / 2$. Under Lorentz transformations they change as

$\lambda_{p, \beta} \rightarrow\left(\Lambda_{R}\right)_{\beta}^{\alpha} \lambda_{p, \alpha}$ with $\quad \Lambda_{R}=e^{(-i \theta-\eta) \cdot \sigma / 2}$

\section{A.3 Four-vectors and bispinors}

We recall from Sect. 3.2, that using the $\tau$ matrices, any fourvector $p_{\mu}$ can be mapped to Hermitian $2 \times 2$-matrices, or bispinors,

$$
\begin{aligned}
& p_{\mu} \tau^{\mu, \dot{\alpha} \beta}=p^{\dot{\alpha} \beta} \leftrightarrow p_{\mu} \tau^{\mu} \\
& =\frac{1}{\sqrt{2}} \not p=\frac{1}{\sqrt{2}}\left(\begin{array}{cc}
p^{0}-p^{3} & i p^{2}-p^{1} \\
-i p^{2}-p^{1} & p^{0}+p^{3}
\end{array}\right)=\frac{1}{\sqrt{2}}\left(\begin{array}{cc}
p^{-} & -p^{\perp^{*}} \\
-p^{\perp} & p^{+}
\end{array}\right),
\end{aligned}
$$

$$
\begin{aligned}
& p_{\mu} \bar{\tau}_{\alpha \dot{\beta}}^{\mu}=\bar{p}_{\alpha \dot{\beta}} \quad \leftrightarrow \quad p_{\mu} \bar{\tau}^{\mu} \\
& =\frac{1}{\sqrt{2}} \bar{p}=\frac{1}{\sqrt{2}}\left(\begin{array}{cc}
p^{0}+p^{3} & p^{1}-i p^{2} \\
p^{1}+i p^{2} & p^{0}-p^{3}
\end{array}\right)=\frac{1}{\sqrt{2}}\left(\begin{array}{c}
p^{+} p^{\perp^{*}} \\
p^{\perp} p^{-}
\end{array}\right),
\end{aligned}
$$

for which we have again used the light-cone coordinates in (A.12). ${ }^{22}$

Raising and lowering spinor indices on the $\tau$ matrices is done by

$\bar{\tau}_{\alpha \dot{\beta}}^{\mu}=\epsilon_{\alpha \gamma} \epsilon_{\dot{\beta} \dot{\eta}} \tau^{\mu, \dot{\eta} \gamma}, \quad \tau^{\mu, \dot{\alpha} \beta}=\epsilon^{\dot{\alpha} \dot{\gamma}} \epsilon^{\beta \eta} \bar{\tau}_{\eta \dot{\gamma}}^{\mu}$

which is easily confirmed for the explicit matrix representations in Eqs. (A.2) and (A.3). The raising and lowering of spinor indices on a four-vector $p_{\mu}$ in the bispinor representation follows by contracting the above with $p_{\mu}$,

$\bar{p}_{\alpha \dot{\beta}}=\epsilon_{\alpha \gamma} \epsilon_{\dot{\beta} \dot{\eta}} p^{\dot{\eta} \gamma}, \quad p^{\dot{\alpha} \beta}=\epsilon^{\dot{\alpha} \dot{\gamma}} \epsilon^{\beta \eta} \bar{p}_{\eta \dot{\gamma}}$,

which may easily be confirmed for the explicit representations in Eqs. (A.25) and (A.26).

We recall from Sect. 3.2 that if a momentum $p$ is massless, i.e. light-like, the corresponding momentum bispinors can be expressed as outer products, or dyads, of Weyl spinors,

$\not p=\mid p]\langle p| \quad$ or $\quad \sqrt{2} p^{\dot{\alpha} \beta}=\tilde{\lambda}_{p}^{\dot{\alpha}} \lambda_{p}^{\beta}, \quad p^{2}=0$,

$\overline{\not p}=|p\rangle\left[p \mid \quad\right.$ or $\quad \sqrt{2} \bar{p}_{\alpha \dot{\beta}}=\lambda_{p, \alpha} \tilde{\lambda}_{p, \dot{\beta}}, \quad p^{2}=0$.

This is easily confirmed for the explicit representations of the Weyl spinors in Eqs. (A.10) and (A.11), comparing to the explicit representation of the momentum bispinors in Eqs. (A.25) and (A.26), and using the conditions for the light-cone coordinates in the massless case, i.e. $p^{+} p^{-}=p^{\perp} p^{\perp^{*}}$ if $p^{2}=0$. If a momentum $p$ is expressed as a linear combination of light-like momenta $p_{i}$, then

$\left.\not p=\sum_{i} c_{i} \mid p_{i}\right]\left\langle p_{i}\right| \quad$ or $\quad \sqrt{2} p^{\dot{\alpha} \beta}=\sum_{i} c_{i} \tilde{\lambda}_{p_{i}}^{\dot{\alpha}} \lambda_{p_{i}}^{\beta}$

for $p=\sum_{i} c_{i} p_{i}$ and $p_{i}^{2}=0$

$\overline{p p}=\sum_{i} c_{i}\left|p_{i}\right\rangle\left[p_{i} \mid \quad\right.$ or $\quad \sqrt{2} \bar{p}_{\alpha \dot{\beta}}=\sum_{i} c_{i} \lambda_{p_{i}, \alpha} \tilde{\lambda}_{p_{i}, \dot{\beta}}$

for $p=\sum_{i} c_{i} p_{i}$ and $p_{i}^{2}=0$

\footnotetext{
22 We repeat, the slash notation here is not to be confused with the Feynman slash notation, denoting contractions with Dirac matrices.
} 
With the above, and the properties of the spinor inner products, it is easy to see that the Weyl spinors obey the Weyl equations,

$$
\begin{aligned}
& p^{\mu} \sigma_{\mu}^{\dot{\alpha} \beta} \lambda_{p, \beta}=\sqrt{2} p^{\dot{\alpha} \beta} \lambda_{p, \beta} \stackrel{p^{2}=0}{=} \tilde{\lambda}_{p}^{\dot{\alpha}} \lambda_{p}^{\beta} \lambda_{p, \beta}=0, \\
& \lambda_{p}^{\alpha} p^{\mu} \bar{\sigma}_{\mu, \alpha \dot{\beta}}=\sqrt{2} \lambda_{p}^{\alpha} \bar{p}_{\alpha \dot{\beta}} \stackrel{p^{2}=0}{=} \lambda_{p}^{\alpha} \lambda_{p, \alpha} \tilde{\lambda}_{p, \dot{\beta}}=0, \\
& p^{\mu} \bar{\sigma}_{\mu, \alpha \dot{\beta}} \tilde{\lambda}_{p}^{\dot{\beta}}=\sqrt{2} \bar{p}_{\alpha \dot{\beta}} \tilde{\lambda}_{p}^{\dot{\beta}} \stackrel{p^{2}=0}{=} \lambda_{p, \alpha} \tilde{\lambda}_{p, \dot{\beta}} \tilde{\lambda}_{p}^{\dot{\beta}}=0, \\
& \tilde{\lambda}_{p, \dot{\alpha}} p^{\mu} \sigma_{\mu}^{\dot{\alpha} \beta}=\sqrt{2} \tilde{\lambda}_{p, \dot{\alpha}} p^{\dot{\alpha} \beta} \stackrel{p^{2}=0}{=} \tilde{\lambda}_{p, \dot{\alpha}} \tilde{\lambda}_{p}^{\dot{\alpha}} \lambda_{p}^{\beta}=0,
\end{aligned}
$$

or

$$
\begin{aligned}
& \left.\left.\not p|p\rangle \stackrel{p^{2}=0}{=}(\mid p]\langle p|\right)|p\rangle=\mid p\right]\langle p p\rangle=0, \\
& \langle p| \bar{p}^{p^{2}=0}={ }^{\prime}\langle p|(|p\rangle[p \mid)=\langle p p\rangle[p \mid=0, \\
& \bar{p} \mid p] \stackrel{p^{2}=0}{=}(|p\rangle[p \mid) \mid p]=|p\rangle[p p]=0, \\
& {\left[p \mid \not p \stackrel{p^{2}=0}{=}[p \mid(\mid p]\langle p|)=[p p]\langle p|=0,\right.}
\end{aligned}
$$

and that for massless particles $i$ and $j$, with $s_{i j}=\left(p_{i}+\right.$ $\left.p_{j}\right)^{2}=2 p_{i} \cdot p_{j}$, the spinor inner products satisfy

$$
\begin{aligned}
s_{i j} & =2 p_{i} \cdot p_{j}=2 p_{i, \mu} p_{j, \nu} \operatorname{Tr}\left(\tau^{\mu} \bar{\tau}^{v}\right)=\sqrt{2} p_{i}^{\dot{\alpha} \beta} \sqrt{2} \bar{p}_{j, \beta \dot{\alpha}} \\
& =\tilde{\lambda}_{i}^{\dot{\alpha}} \lambda_{i}^{\beta} \lambda_{j, \beta} \tilde{\lambda}_{j, \dot{\alpha}}=\lambda_{i}^{\beta} \lambda_{j, \beta} \tilde{\lambda}_{j, \dot{\alpha}} \tilde{\lambda}_{i}^{\dot{\alpha}}=\langle i j\rangle[j i] .
\end{aligned}
$$

Using Eqs. (A.29) and (A.30), we have

$\left[k\left|\not p_{i}\right| l\right\rangle=[k i]\langle i l\rangle, \quad\left\langle k\left|\overline{\not p}_{i}\right| l\right]=\langle k i\rangle[i l]$ for $p_{i}^{2}=0$.

Assuming a set of outgoing massless external momenta $p_{i}$, and using momentum conservation, i.e. $\sum_{i} p_{i}=0$, gives

$$
\begin{aligned}
& \sum_{i}[j i]\langle i k\rangle=\sum_{i \neq j, k}[j i]\langle i k\rangle=0, \\
& \sum_{i}\langle j i\rangle[i k]=\sum_{i \neq j, k}\langle j i\rangle[i k]=0 .
\end{aligned}
$$

Using $\lambda_{i}^{\alpha} \bar{\tau}_{\alpha \dot{\beta}}^{\mu} \tilde{\lambda}_{j}^{\dot{\beta}}=\tilde{\lambda}_{j, \dot{\delta}} \tau^{\mu, \dot{\delta} \gamma} \lambda_{i, \gamma}$ (see (4.8)), or equivalently $\left\langle i\left|\bar{\tau}^{\mu}\right| j\right]=\left[j\left|\tau^{\mu}\right| i\right\rangle\left(\right.$ see (4.9)), and $\tau_{\mu}^{\dot{\alpha} \beta} \bar{\tau}_{\gamma \dot{\eta}}^{\mu}=\delta_{\gamma}^{\beta}{ }^{\beta}{ }_{\dot{\eta}}^{\dot{\alpha}}$ (see (4.3)), this implies

$$
\begin{aligned}
{\left[i\left|\tau^{\mu}\right| j\right\rangle\left[k\left|\tau_{\mu}\right| l\right\rangle } & =[k i]\langle j l\rangle, \\
\left\langle i\left|\bar{\tau}^{\mu}\right| j\right]\left\langle k\left|\bar{\tau}_{\mu}\right| l\right] & =[j l]\langle k i\rangle, \\
{\left[i\left|\tau^{\mu}\right| j\right\rangle\left\langle k\left|\bar{\tau}_{\mu}\right| l\right] } & =[i l]\langle k j\rangle .
\end{aligned}
$$

For completeness, we also repeat the expressions for the polarization vectors of massless vector bosons from Sect. 3.3. The polarization vectors can be written in terms of Weyl spinors and $\tau$ matrices,

$\epsilon_{-}^{\mu}\left(p_{i}, r\right)=\frac{\lambda_{i}^{\alpha} \bar{\tau}_{\alpha \dot{\beta}^{\mu}}^{\mu} \tilde{\lambda}_{r}^{\dot{\beta}}}{\tilde{\lambda}_{i, \dot{\gamma}} \tilde{\lambda}_{r}^{\dot{\gamma}}}=\frac{\left\langle i\left|\bar{\tau}^{\mu}\right| r\right]}{[i r]}$,

$\epsilon_{+}^{\mu}\left(p_{i}, r\right)=\frac{\lambda_{r}^{\alpha} \bar{\tau}_{\alpha \dot{\beta}}^{\mu} \tilde{\lambda}_{i}^{\dot{\beta}}}{\lambda_{r}^{\gamma} \lambda_{i, \gamma}}=\frac{\left\langle r\left|\bar{\tau}^{\mu}\right| i\right]}{\langle r i\rangle}$,

$\epsilon_{-}^{\mu}\left(p_{i}, r\right)=\frac{\tilde{\lambda}_{r, \dot{\alpha}} \tau^{\mu, \dot{\alpha} \beta} \lambda_{i, \beta}}{\tilde{\lambda}_{i, \dot{\gamma}} \tilde{\lambda}_{r}^{\dot{\gamma}}}=\frac{\left[r\left|\tau^{\mu}\right| i\right\rangle}{[i r]}$,

$\epsilon_{+}^{\mu}\left(p_{i}, r\right)=\frac{\tilde{\lambda}_{i, \dot{\alpha}} \tau^{\mu, \dot{\alpha} \beta} \lambda_{r, \beta}}{\lambda_{r}^{\gamma} \lambda_{i, \gamma}}=\frac{\left[i\left|\tau^{\mu}\right| r\right\rangle}{\langle r i\rangle}$,

where $p_{i}$ is the vector boson momentum and $r$ is an arbitrary, light-like reference momentum satisfying $p_{i} \cdot r \neq 0$. To get from (A.41) to (A.42) we have used $\lambda_{i}^{\alpha} \bar{\tau}_{\alpha \dot{\beta}}^{\mu} \tilde{\lambda}_{j}^{\dot{\beta}}=$ $\tilde{\lambda}_{j, \delta} \tau^{\mu, \dot{\delta} \gamma} \lambda_{i, \gamma}$ (see (4.8)). With the above, and the properties of massless spinors and bispinors, as stated before, it is easily confirmed that $\left(\epsilon_{-}^{\mu}\left(p_{i}, r\right)\right)^{*}=\epsilon_{+}^{\mu}\left(p_{i}, r\right)$, as well as $\epsilon_{ \pm}^{\mu}\left(p_{i}, r\right) p_{i, \mu}=0$ and $\epsilon_{ \pm}^{\mu}\left(p_{i}, r\right) r_{\mu}=0$. Contracting (A.41) with $\tau$, (A.42) with $\bar{\tau}$, and using (A.7), we may write the polarization vectors in the bispinor representation in terms of outer products, or dyads, of Weyl spinors, ${ }^{23}$

$$
\begin{gathered}
\epsilon_{-}^{\dot{\beta} \alpha}\left(p_{i}, r\right)=\epsilon_{-}^{\mu}\left(p_{i}, r\right) \tau_{\mu}^{\dot{\beta} \alpha}=\frac{\tilde{\lambda}_{r}^{\dot{\beta}} \lambda_{i}^{\alpha}}{\tilde{\lambda}_{i, \dot{\gamma}} \tilde{\lambda}_{r}^{\dot{\gamma}}}=\frac{\mid r]\langle i|}{[i r]}, \\
\epsilon_{+}^{\dot{\beta} \alpha}\left(p_{i}, r\right)=\epsilon_{+}^{\mu}\left(p_{i}, r\right) \tau_{\mu}^{\dot{\beta} \alpha}=\frac{\tilde{\lambda}_{i}^{\dot{\beta}} \lambda_{r}^{\alpha}}{\lambda_{r}^{\gamma} \lambda_{i, \gamma}}=\frac{\mid i]\langle r|}{\langle r i\rangle}, \\
\bar{\epsilon}_{-, \beta \dot{\alpha}}\left(p_{i}, r\right)=\epsilon_{-}^{\mu}\left(p_{i}, r\right) \bar{\tau}_{\mu, \beta \dot{\alpha}}=\frac{\lambda_{i, \beta} \tilde{\lambda}_{r, \dot{\alpha}}}{\tilde{\lambda}_{i, \dot{\gamma}} \tilde{\lambda}_{r}^{\dot{\gamma}}}=\frac{|i\rangle[r \mid}{[i r]}, \\
\bar{\epsilon}_{+, \beta \dot{\alpha}}\left(p_{i}, r\right)=\epsilon_{+}^{\mu}\left(p_{i}, r\right) \bar{\tau}_{\mu, \beta \dot{\alpha}}=\frac{\lambda_{r, \beta} \tilde{\lambda}_{i, \dot{\alpha}}}{\lambda_{r}^{\gamma} \lambda_{i, \gamma}}=\frac{|r\rangle[i \mid}{\langle r i\rangle} .
\end{gathered}
$$

A.4 Tables with QED and QCD conventions and Feynman rules

We collect here spinor notation conventions, as well as chirality-flow Feynman rules for QED and QCD.

\footnotetext{
23 We could have equally well contracted (A.41) with $\bar{\tau}$ and (A.42) with $\tau$, using Eqs. (A.8) and (A.9), respectively.
} 
Table 1 The QED "Rosetta Stone" translating the chirality-flow notation to widely-used spinor-helicity notations. For more information see Sects. 3.1 (external fermions), 3.3 (external vector bosons), 5.1 (vertices) and 5.2 (propagators)

\begin{tabular}{|c|c|c|c|c|c|}
\hline (Outgoing) Species & Dirac & Index & Bra-ket & Feynman & Chirality-flow \\
\hline $\begin{array}{l}\text { right-chiral fermion } \\
\text { right-chiral anti-fermion }\end{array}$ & $\begin{array}{c}\bar{u}\left(p_{i}\right) P_{R}=\left(0,\left(u_{L}\left(p_{i}\right)\right)^{\dagger}\right) \\
P_{R} v\left(p_{j}\right)=\left(\begin{array}{c}0 \\
v_{R}\left(p_{j}\right)\end{array}\right)\end{array}$ & $\begin{array}{l}\lambda_{i}^{\alpha} \\
\lambda_{j, \alpha}\end{array}$ & $\begin{array}{l}\langle i| \\
|j\rangle\end{array}$ & ${ }_{-}^{i}$ & - \\
\hline $\begin{array}{l}\text { left-chiral fermion } \\
\text { left-chiral anti-fermion }\end{array}$ & $\begin{array}{c}\bar{u}\left(p_{i}\right) P_{L}=\left(\left(u_{R}\left(p_{i}\right)\right)^{\dagger}, 0\right) \\
P_{L} v\left(p_{j}\right)=\left(\begin{array}{c}v_{L}\left(p_{j}\right) \\
0\end{array}\right)\end{array}$ & $\begin{array}{l}\tilde{\lambda}_{i, \dot{\alpha}} \\
\tilde{\lambda}_{j}^{\dot{\alpha}}\end{array}$ & $\begin{array}{l}{[i \mid} \\
\mid j]\end{array}$ & & O. \\
\hline $\begin{array}{l}\text { negative-helicity photon } \\
\text { positive-helicity photon }\end{array}$ & $\begin{array}{l}\epsilon_{-}^{\mu}\left(p_{i}, r\right) \\
\epsilon_{+}^{\mu}\left(p_{i}, r\right)\end{array}$ & $\begin{array}{l}\frac{\lambda_{i}^{\alpha} \bar{\tau}_{\alpha \dot{\beta}}^{\mu} \tilde{\lambda}_{r}^{\dot{\beta}}}{[i r]} \text { or } \frac{\tilde{\lambda}_{r, \dot{\beta}}{ }^{\mu, \dot{\beta} \alpha} \lambda_{i, \alpha}}{[i r]} \\
\frac{\lambda_{r}^{\beta} \bar{\tau}_{\beta \dot{\alpha}}^{\mu} \tilde{\lambda}_{i}^{\dot{\alpha}}}{\langle r i\rangle} \text { or } \frac{\tilde{\lambda}_{i, \dot{\alpha}} \tau^{\mu, \dot{\alpha} \beta} \lambda_{r, \beta}}{\langle r i\rangle}\end{array}$ & $\begin{array}{l}\frac{\left\langle i\left|\overline{\mid}^{\mu}\right| r\right]}{[i r]} \text { or } \frac{\left[r\left|\tau^{\mu}\right| i\right\rangle}{[i r]} \\
\frac{\left\langle r\left|\overline{\mid}^{\mu}\right| i\right]}{\langle r i\rangle} \text { or } \frac{\left[i||^{\mu}|r\rangle\right.}{\langle r i\rangle}\end{array}$ & $\mathrm{mm}_{-}^{i}$ & $\begin{array}{l}\frac{1}{[i r]} \bigcirc \cdots{ }_{i} \text { or } \frac{1}{[i r]} \bigcirc \cdots{ }_{r}^{r} \text { or } \frac{1}{\langle r i\rangle} \bigcirc \cdots{ }_{r}^{r} \\
\frac{1}{\langle r i\rangle} \bigcirc \cdots \cdots\end{array}$ \\
\hline Vertices & Dirac & Index & Bra-ket & Feynman & Chirality-flow \\
\hline fermion-photon & $i e Q_{f} \gamma^{\mu}$ & & & & \\
\hline fermion-photon & $i e Q_{f} \gamma^{\mu}$ & $i e Q_{f} \sqrt{2} \bar{\tau}_{\alpha \dot{\beta}}^{\mu}$ & $i e Q_{f} \sqrt{2} \bar{\tau}_{\alpha \dot{\beta}}^{\mu}$ & & $i e Q_{f} \sqrt{2}$ \\
\hline Propagators & Dirac & Index & Bra-ket & Feynman & Chirality-flow \\
\hline fermion & $\frac{i}{p_{\mu} \gamma^{\mu}}=\frac{i p_{\mu} \gamma^{\mu}}{p^{2}}$ & $i \frac{\tilde{\lambda}_{p}^{\alpha} \lambda_{p}^{\beta}}{p^{2}}$ & $i \frac{|p|\langle p|}{p^{2}}$ & $\frac{p}{\stackrel{\alpha}{\longleftarrow}}$ & $\frac{i}{p^{2}}-\dot{\alpha}_{-}^{l}$ \\
\hline fermion & $\frac{i}{p_{\mu} \gamma^{\mu}}=\frac{i p_{\mu} \gamma^{\mu}}{p^{2}}$ & $i \frac{\lambda_{p, \alpha} \tilde{\lambda}_{p, \hat{\beta}}}{p^{2}}$ & $i \frac{|p\rangle[p \mid}{p^{2}}$ & $\frac{p}{\alpha} \dot{\beta}$ & $\frac{i}{p^{2}} \stackrel{\alpha}{p_{\beta}^{\beta}} \longrightarrow-$ \\
\hline photon & $-i \frac{g_{\mu \nu}}{p^{2}}$ & $-i \frac{g_{\mu \nu}}{p^{2}}$ & $-i \frac{g_{\mu \nu}}{p^{2}}$ & $\mu \stackrel{p}{\sim} \sim \nu$ & $-\frac{i}{p^{2}} \longrightarrow \ldots \ldots$ \\
\hline
\end{tabular}

Table 2 The QCD "Rosetta Stone" translating the chirality-flow notation to widely-used spinor-helicity notations. $V_{3}^{\mu_{1} \mu_{2} \mu_{2}} \equiv V_{3}^{\mu_{1} \mu_{2} \mu_{2}}\left(p_{1}, p_{2}, p_{3}\right)$ is given in (5.7). For more information, in particular on the various forms of the four-gluon vertex, see Sect. 5.1 (vertices) and 5.2 (propagators)

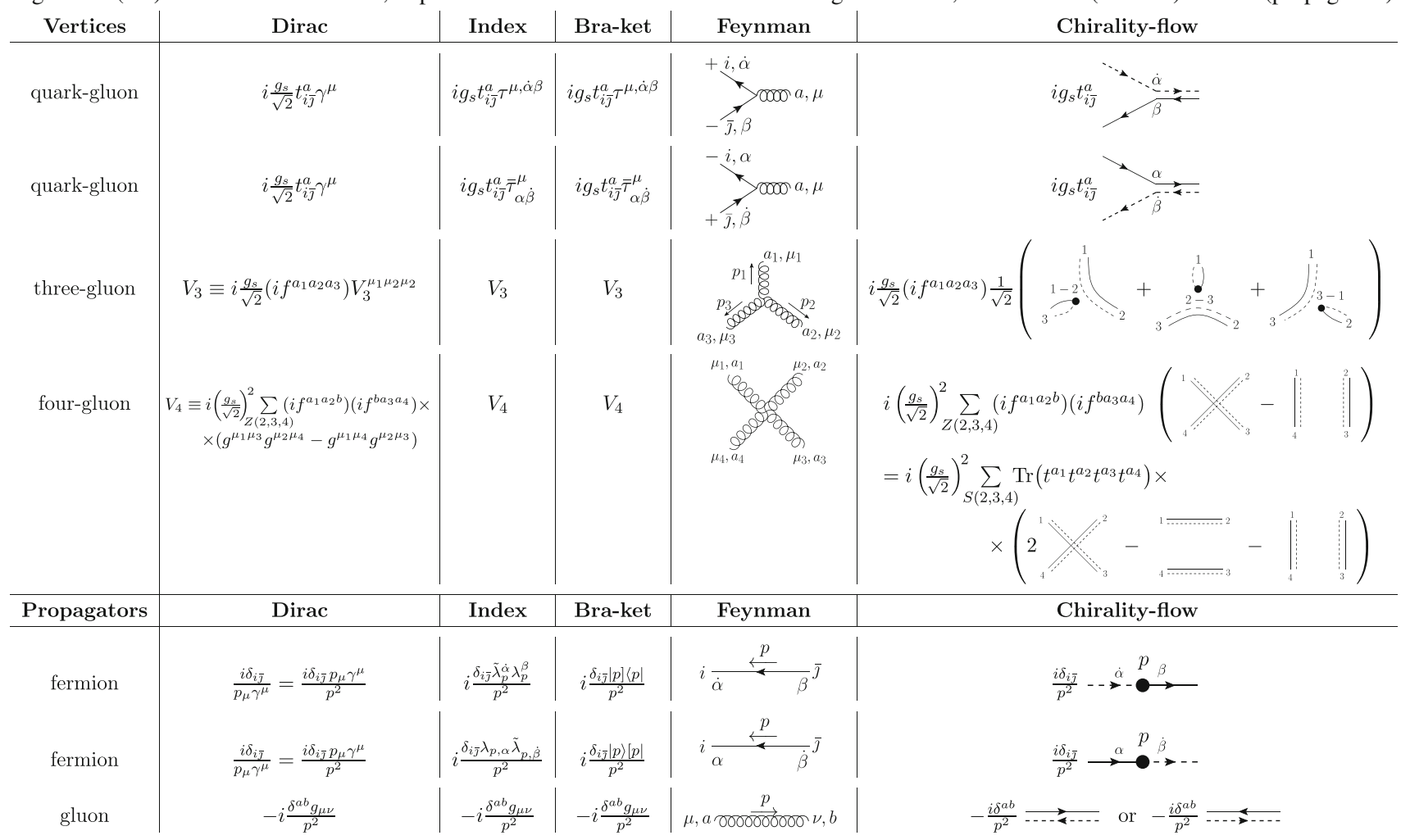




\section{References}

1. A. Macfarlane, A. Sudbery, P. Weisz, On Gell-Mann's $\lambda$-matrices, $d$ - and $f$-tensors, octets, and parametrizations of SU(3). Commun. Math. Phys. 11, 77 (1968)

2. J.E. Paton, H.-M. Chan, Generalized Veneziano model with isospin. Nucl. Phys. B 10, 516 (1969). https://doi.org/10.1016/ 0550-3213(69)90038-8

3. G. Hooft, A planar diagram theory for strong interactions. Nucl. Phys. B 72, 461 (1974). https://doi.org/10.1016/ 0550-3213(74)90154-0

4. P. Cvitanović, Group theory for Feynman diagrams in non-Abelian gauge theories. Phys. Rev. D 14, 1536 (1976)

5. P. Butera, G.M. Cicuta, M. Enriotti, Group weight and vanishing graphs. Phys. Rev. D 21, 972 (1980). https://doi.org/10.1103/ PhysRevD.21.972

6. P. Cvitanovic, P.G. Lauwers, P.N. Scharbach, Gauge invariance structure of quantum chromodynamics. Nucl. Phys. B 186, 165 (1981). https://doi.org/10.1016/0550-3213(81)90098-5

7. F.A. Berends, W. Giele, The six gluon process as an example of Weyl-Van Der Waerden spinor calculus. Nucl. Phys. B 294, 700 (1987). https://doi.org/10.1016/0550-3213(87)90604-3

8. F.A. Berends, W.T. Giele, Recursive calculations for processes with n gluons. Nucl. Phys. B 306, 759 (1988). https://doi.org/10.1016/ 0550-3213(88)90442-7

9. M.L. Mangano, S.J. Parke, Z. Xu, Duality and multi-gluon scattering. Nucl. Phys. B 298, 653 (1988). https://doi.org/10.1016/ 0550-3213(88)90001-6

10. M.L. Mangano, The color structure of gluon emission. Nucl. Phys. B 309, 461 (1988). https://doi.org/10.1016/ 0550-3213(88)90453-1

11. D. Zeppenfeld, Diagonalization of color factors. Int. J. Mod. Phys. A 3, 2175 (1988). https://doi.org/10.1142/S0217751X88000916

12. D.A. Kosower, Color factorization for fermionic amplitudes. Nucl. Phys. B 315, 391 (1989). https://doi.org/10.1016/ 0550-3213(89)90361-1

13. F.A. Berends, W.T. Giele, H. Kuijf, Exact expressions for processes involving a vector boson and up to five partons. Nucl. Phys. B 321, 39 (1989). https://doi.org/10.1016/0550-3213(89)90242-3

14. F.A. Berends, W.T. Giele, Multiple soft gluon radiation in parton processes. Nucl. Phys. B 313, 595 (1989). https://doi.org/10.1016/ 0550-3213(89)90398-2

15. F.A. Berends, W.T. Giele, H. Kuijf, Exact and approximate expressions for multi-gluon scattering. Nucl. Phys. B 333, 120 (1990). https://doi.org/10.1016/0550-3213(90)90225-3

16. Z. Bern, D.A. Kosower, Color decomposition of one loop amplitudes in gauge theories. Nucl. Phys. B 362, 389 (1991). https://doi. org/10.1016/0550-3213(91)90567-H

17. Z. Bern, L.J. Dixon, D.A. Kosower, One loop corrections to two quark three gluon amplitudes. Nucl. Phys. B 437, 259 (1995). https://doi.org/10.1016/0550-3213(94)00542-M. arXiv:hep-ph/9409393

18. V. Del Duca, L.J. Dixon, F. Maltoni, New color decompositions for gauge amplitudes at tree and loop level. Nucl. Phys. B 571, 51 (2000). https://doi.org/10.1016/S0550-3213(99)00809-3. arXiv:hep-ph/9910563

19. F. Maltoni, K. Paul, T. Stelzer, S. Willenbrock, Color flow decomposition of QCD amplitudes. Phys. Rev. D 67, 014026 (2003). https://doi.org/10.1103/PhysRevD.67.014026. arXiv:hep-ph/0209271

20. S. Weinzierl, Automated computation of spin- and colourcorrelated Born matrix elements. Eur. Phys. J. C 45, 745 (2006). https://doi.org/10.1140/epjc/s2005-02467-6. arXiv:hep-ph/0510157
21. R.K. Ellis, W.T. Giele, Z. Kunszt, K. Melnikov, G. Zanderighi, Oneloop amplitudes for $W^{+} 3$ jet production in hadron collisions. JHEP 01, 012 (2009). https://doi.org/10.1088/1126-6708/2009/01/012. arXiv:0810.2762

22. M. Sjodahl, Color evolution of $2 \rightarrow 3$ processes. JHEP 12, 083 (2008). https://doi.org/10.1088/1126-6708/2008/12/083. arXiv:0807.0555

23. M. Sjodahl, Color structure for soft gluon resummation: a general recipe. JHEP 0909, 087 (2009). https://doi.org/10.1088/ 1126-6708/2009/09/087. arXiv:0906.1121

24. H. Ita, K. Ozeren, Colour decompositions of multi-quark one-loop QCD amplitudes. JHEP 02, 118 (2012). https://doi.org/10.1007/ JHEP02(2012)118. arXiv:1111.4193

25. S. Keppeler, M. Sjodahl, Orthogonal multiplet bases in SU(Nc) color space. JHEP 09, 124 (2012). https://doi.org/10.1007/ JHEP09(2012)124. arXiv:1207.0609

26. C. Reuschle, Numerical precision calculations for LHC physics. https://publications.ub.uni-mainz.de/theses/frontdoor.php? source_opus=3389, Ph.D. thesis, Mainz, Johannes Gutenberg Universität, (2013)

27. T. Schuster, Color ordering in QCD. Phys. Rev. D 89, 105022 (2014). https://doi.org/10.1103/PhysRevD.89.105022. arXiv: 1311.6296

28. C. Reuschle, S. Weinzierl, Decomposition of one-loop QCD amplitudes into primitive amplitudes based on shuffle relations. Phys. Rev. D 88, 105020 (2013). https://doi.org/10.1103/PhysRevD.88. 105020. arXiv: 1310.0413

29. Y.-J. Du, M. Sjödahl, J. Thorén, Recursion in multiplet bases for tree-level MHV gluon amplitudes. JHEP 05, 119 (2015). https:// doi.org/10.1007/JHEP05(2015)119. arXiv:1503.00530

30. M. Sjodahl, J. Thorén, Decomposing color structure into multiplet bases. JHEP 09, 055 (2015). https://doi.org/10.1007/ JHEP09(2015)055. arXiv:1507.03814

31. M. Sjodahl, J. Thorén, QCD multiplet bases with arbitrary parton ordering. JHEP 11, 198 (2018). https://doi.org/10.1007/ JHEP11(2018)198. arXiv:1809.05002

32. W. Kilian, T. Ohl, J. Reuter, C. Speckner, QCD in the color-flow representation. JHEP 1210, 022 (2012). https://doi.org/10.1007/ JHEP10(2012)022. arXiv:1206.3700 [hep-ph]

33. P. De Causmaecker, R. Gastmans, W. Troost, T.T. Wu, Multiple Bremsstrahlung in gauge theories at high-energies. 1. General formalism for quantum electrodynamics. Nucl. Phys. B 206, 53 (1982). https://doi.org/10.1016/0550-3213(82)90488-6

34. F.A. Berends, R. Kleiss, P. De Causmaecker, R. Gastmans, T.T. Wu, Single Bremsstrahlung processes in gauge theories. Phys. Lett. B 103, 124 (1981). https://doi.org/10.1016/0370-2693(81)90685-7

35. F.A. Berends, R. Kleiss, P. De Causmaecker, R. Gastmans, W. Troost, T.T. Wu, Multiple Bremsstrahlung in gauge theories at highenergies. 2. Single Bremsstrahlung. Nucl. Phys. B 206, 61 (1982). https://doi.org/10.1016/0550-3213(82)90489-8

36. P. De Causmaecker, R. Gastmans, W. Troost, T.T. Wu, Helicity amplitudes for massless QED. Phys. Lett. B 105, 215 (1981). https://doi.org/10.1016/0370-2693(81)91025-X

37. CALKUL collaboration, F.A. Berends, R. Kleiss, P. de Causmaecker, R. Gastmans, W. Troost, T.T. Wu, Multiple Bremsstrahlung in gauge theories at high-energies. 3. Finite mass effects in collinear photon Bremsstrahlung. Nucl. Phys. B239 382 (1984). https://doi.org/10.1016/0550-3213(84)90254-2

38. R. Kleiss, The cross-section for $e^{+} e^{-} \rightarrow e^{+} e^{-} e^{+} e^{-}$. Nucl. Phys. B 241, 61 (1984). https://doi.org/10.1016/0550-3213(84)90197-4

39. F.A. Berends, P.H. Daverveldt, R. Kleiss, Complete lowest order calculations for four lepton final states in electron-positron collisions. Nucl. Phys. B 253, 441 (1985). https://doi.org/10.1016/ 0550-3213(85)90541-3 
40. J.F. Gunion, Z. Kunszt, Four jet processes: gluon-gluon scattering to nonidentical quark-anti-quark pairs. Phys. Lett. B 159, 167 (1985). https://doi.org/10.1016/0370-2693(85)90879-2

41. J.F. Gunion, Z. Kunszt, Improved analytic techniques for tree graph calculations and the $\mathrm{G} g \mathrm{q}$ anti-q lepton anti-lepton subprocess. Phys. Lett. B 161, 333 (1985). https://doi.org/10.1016/ 0370-2693(85)90774-9

42. R. Kleiss, W.J. Stirling, Spinor techniques for calculating $p$ anti-p $\longrightarrow$ W+- / Z0 + jets. Nucl. Phys. B 262, 235 (1985). https://doi. org/10.1016/0550-3213(85)90285-8

43. K. Hagiwara, D. Zeppenfeld, Helicity amplitudes for heavy lepton production in e+ e- annihilation. Nucl. Phys. B 274, 1 (1986). https://doi.org/10.1016/0550-3213(86)90615-2

44. R. Kleiss, Hard Bremsstrahlung amplitudes for $e^{+} e^{-}$collisions with polarized beams at LEP/SLC energies. Z. Phys. C 33, 433 (1987). https://doi.org/10.1007/BF01552550

45. R. Kleiss, W.J. Stirling, Cross-sections for the production of an arbitrary number of photons in electron-positron annihilation. Phys. Lett. B 179, 159 (1986). https://doi.org/10.1016/ 0370-2693(86)90454-5

46. Z. Xu, D.-H. Zhang, L. Chang, Helicity amplitudes for multiple Bremsstrahlung in massless nonabelian gauge theories. Nucl. Phys. B 291, 392 (1987). https://doi.org/10.1016/ 0550-3213(87)90479-2

47. CALKUL collaboration, R. Gastmans, F.A. Berends, D. Danckaert, P. De Causmaecker, R. Kleiss, W. Troost et al., New techniques and results in gauge theory calculations, in Electroweak effects at highenergies. Proceedings, 1st Europhysics study conference, Erice, Italy, February 1-12, 1983, pp. 599-609 (1987)

48. C. Schwinn, S. Weinzierl, Scalar diagrammatic rules for Born amplitudes in QCD. JHEP 05, 006 (2005). https://doi.org/10.1088/ 1126-6708/2005/05/006. arXiv:hep-th/0503015

49. G.R. Farrar, F. Neri, How to Calculate 35640 O $\left(\alpha^{5}\right)$ Feynman diagrams in less than an hour. Phys. Lett. B 130, 109 (1983). https://doi.org/10.1016/0370-2693(85)90526-X. https:// doi.org/10.1016/0370-2693(83)91074-2

50. S. Dittmaier, Full O(alpha) radiative corrections to high-energy Compton scattering. Nucl. Phys. B 423, 384 (1994). https://doi. org/10.1016/0550-3213(94)90139-2. arXiv:hep-ph/9311363

51. S. Dittmaier, Weyl-van der Waerden formalism for helicity amplitudes of massive particles. Phys. Rev. D 59, 016007 (1998). https:// doi.org/10.1103/PhysRevD.59.016007. arXiv:hep-ph/9805445

52. M.T. Grisaru, H.N. Pendleton, Some properties of scattering amplitudes in supersymmetric theories. Nucl. Phys. B 124, 81 (1977). https://doi.org/10.1016/0550-3213(77)90277-2

53. S.J. Parke, T.R. Taylor, Perturbative QCD utilizing extended supersymmetry. Phys. Lett. B 157, 81 (1985). https://doi.org/10.1016/ 0370-2693(85)91216-X

54. S.J. Parke, T.R. Taylor, An amplitude for $n$ gluon scattering. Phys. Rev. Lett. 56, 2459 (1986). https://doi.org/10.1103/PhysRevLett. 56.2459

55. C. Schwinn, S. Weinzierl, SUSY ward identities for multigluon helicity amplitudes with massive quarks. JHEP 03, 030 (2006). https://doi.org/10.1088/1126-6708/2006/03/030. arXiv:hep-th/0602012

56. P. Cvitanović, Group theory: Birdtracks, Lie's, and exceptional groups (Princeton University Press, Princeton, 2008)

57. A.D. Kennedy, Spinography: diagrammatic methods for spinors in Feynman diagrams. Phys. Rev. D 26, 1936 (1982). https://doi.org/ 10.1103/PhysRevD.26.1936

58. M.L. Mangano, S.J. Parke, Multiparton amplitudes in gauge theories. Phys. Rep. 200, 301 (1991). https://doi.org/10.1016/ 0370-1573(91)90091-Y. arXiv:hep-th/0509223

59. R. Gastmans, T.T. Wu, The ubiquitous photon: helicity method for QED and QCD. Int. Ser. Monogr. Phys. 80, 1 (1990)
60. L.J. Dixon, Calculating scattering amplitudes efficiently, in QCD and beyond. Proceedings, Theoretical Advanced Study Institute in Elementary Particle Physics, TASI-95, Boulder, USA, June 4-30, 1995, pp. 539-584 (1996). arXiv:hep-ph/9601359. http://www-public.slac.stanford.edu/sciDoc/docMeta.aspx? slacPubNumber=SLAC-PUB-7106

61. S. Weinzierl, Automated calculations for multi-leg processes. PoS ACAT, 005 (2007).https://doi.org/10.22323/1.050.0005. arXiv:0707.3342

62. H.K. Dreiner, H.E. Haber, S.P. Martin, Two-component spinor techniques and Feynman rules for quantum field theory and supersymmetry. Phys. Rep. 494, 1 (2010). https://doi.org/10.1016/j. physrep.2010.05.002. arXiv:0812.1594

63. R.K. Ellis, Z. Kunszt, K. Melnikov, G. Zanderighi, One-loop calculations in quantum field theory: from Feynman diagrams to unitarity cuts. Phys. Rep. 518, 141 (2012). https://doi.org/10.1016/j. physrep.2012.01.008. arXiv:1105.4319

64. M.E. Peskin, Simplifying Multi-Jet QCD Computation, in Proceedings, 13th Mexican School of Particles and Fields (MSPF 2008): San Carlos, Sonora, Mexico, October 2-11, 2008, (2011). arXiv:1101.2414. http://www-public.slac.stanford.edu/ sciDoc/docMeta.aspx?slacPubNumber=SLAC-PUB- 14352

65. H. Elvang, Y.-T. Huang, Scattering amplitudes. arXiv:1308.1697

66. L.J. Dixon, A brief introduction to modern amplitude methods, in Proceedings, 2012 European School of High-Energy Physics (ESHEP 2012): La Pommeraye, Anjou, France, June 06-19, 2012, pp. 31-67, (2014). https://doi.org/10.5170/CERN-2014-008.31. arXiv: 1310.5353

67. Z. Nagy, D.E. Soper, Parton showers with quantum interference. JHEP 09, 114 (2007). https://doi.org/10.1088/1126-6708/2007/ 09/114. arXiv:0706.0017

68. S. Plätzer, M. Sjodahl, Subleading $N_{c}$ improved parton showers. JHEP 1207, 042 (2012). https://doi.org/10.1007/ JHEP07(2012)042. arXiv: 1201.0260

69. M. Sjödahl, ColorMath: a package for color summed calculations in SU(Nc). Eur. Phys. J. C 73, 2310 (2013). https://doi.org/10.1140/ epjc/s10052-013-2310-4. arXiv:1211.2099

70. M. Sjodahl, ColorFull: a C++ library for calculations in SU(Nc) color space. Eur. Phys. J. C 75, 236 (2015). https://doi.org/10.1140/ epjc/s10052-015-3417-6. arXiv:1412.3967

71. J. Alcock-Zeilinger, H. Weigert, Transition operators. J. Math. Phys. 58, 051703 (2017). https://doi.org/10.1063/1.4983479. arXiv: 1610.08802

72. A. Kyrieleis, M.H. Seymour, The colour evolution of the process $q q \rightarrow q q g$. JHEP 01, 085 (2006). arXiv:hep-ph/0510089

73. Y.L. Dokshitzer, G. Marchesini, Soft gluons at large angles in hadron collisions. JHEP 01, 007 (2006). arXiv:hep-ph/0509078

74. S. Honeywell, S. Quackenbush, L. Reina, C. Reuschle, NLOX, a one-loop provider for Standard Model processes. arXiv: 1812.11925

75. G. Cullen et al., GOSAM-2.0: a tool for automated one-loop calculations within the Standard Model and beyond. Eur. Phys. J. C 74, 3001 (2014). https://doi.org/10.1140/epjc/s10052-014-3001-5. arXiv: 1404.7096

76. J. Alwall, R. Frederix, S. Frixione, V. Hirschi, F. Maltoni, O. Mattelaer et al., The automated computation of tree-level and nextto-leading order differential cross sections, and their matching to parton shower simulations. JHEP 07, 079 (2014). https://doi.org/ 10.1007/JHEP07(2014)079. arXiv:1405.0301 